\title{
Liver fat storage is controlled by HNF4a through induction of lipophagy and is reversed by a potent HNF4a agonist
}

\author{
Seung-Hee Lee ${ }^{1}$, Vimal Veeriah ${ }^{1}$ and Fred Levine (1)
}

\begin{abstract}
We report the discovery of strong HNF4a agonists and their use to uncover a previously unknown pathway by which HNF4a controls the level of fat storage in the liver. This involves the induction of lipophagy by dihydroceramides, the synthesis and secretion of which is controlled by genes induced by HNF4a. The HNF4a activators are N-trans caffeoyltyramine (NCT) and N-trans feruloyltyramine (NFT), which are structurally related to the known drugs alverine and benfluorex, which we previously showed to be weak HNF4a activators. In vitro, NCT and NFT induced fat clearance from palmitate-loaded cells. In DIO mice, NCT led to recovery of hepatic HNF4a expression and reduction of steatosis. Mechanistically, increased dihydroceramide production and action downstream of HNF4a occurred through increased expression of HNF4a downstream genes, including SPNS2 and CYP26A1. NCT was completely nontoxic at the highest dose administered and so is a strong candidate for an NAFLD therapeutic.
\end{abstract}

\section{Introduction}

Fatty liver disease is a major cause of morbidity and mortality ${ }^{1-3}$. Excessive hepatic fat storage secondary to obesity causes hepatocyte dysfunction, termed nonalcoholic fatty liver disease (NAFLD). NAFLD progresses in many cases to non-alcoholic steatotic hepatitis (NASH), characterized by inflammation, fibrosis and hepatocyte death. In some individuals, this progresses further to cirrhosis and organ failure. Obesity-associated liver disease is a leading cause of liver transplantation ${ }^{2}$.

Fat is stored primarily in adipocytes and hepatocytes. Control of fat storage has been studied extensively in adipocytes $^{4}$, where lipase activity is tightly regulated, including by insulin-mediated repression of hormonesensitive lipase so that fat is stored in the fed state when insulin levels are high and released under starvation conditions when insulin is low. In contrast, the control of fat storage in the liver has been poorly understood.

Correspondence: Fred Levine (flevine@sbpdiscovery.org)

${ }^{1}$ SBP Medical Discovery Institute, La Jolla, CA, USA

These authors contributed equally: Seung-Hee Lee, Vimal Veeriah

Edited by A. Finazzi-Agrò
Hepatic fat is an important source of fatty acids that can be mobilized to supply energy ${ }^{5}$. As in the adipocyte, hepatic fat is stored in lipid droplets, which are similar in most respects to thosein adipocytes ${ }^{6}$. However, in contrast to the adipocyte, the liver does not express hormone sensitive lipase and so a different but heretofore unknown mechanism must be used to control fat mobilization from hepatic lipid droplets. In particular, the mechanisms underlying the pathological fat storage that is the hallmark of NAFLD has not been clear. The mechanism by which fat storage in the liver is regulated must include two principal components: a mechanism to sense the amount of fat and a mechanism to control the amount of fat stored in hepatic lipid droplets. Because understanding the control of fat storage in the liver is critical to the development of therapeutics for fatty liver disease, there has been great interest in this area ${ }^{6}$.

Some time ago, we developed a cell based phenotypic screen for compounds that modulate the activity of a human insulin promoter-GFP transgene and used it to screen different compound libraries ${ }^{7-11}$. An important discovery is that our assay is highly sensitive to the level of

\section{(c) The Author(s) 2021}

(c) (i) Open Access This article is licensed under a Creative Commons Attribution 4.0 International License, which permits use, sharing, adaptation, distribution and reproduction cc) in any medium or format, as long as you give appropriate credit to the original author(s) and the source, provide a link to the Creative Commons license, and indicate if changes were made. The images or other third party material in this article are included in the article's Creative Commons license, unless indicated otherwise in a credit line to the material. If material is not included in the article's Creative Commons license and your intended use is not permitted by statutory regulation or exceeds the permitted use, you will need to obtain permission directly from the copyright holder. To view a copy of this license, visit http://creativecommons.org/licenses/by/4.0/. 
activity of the nuclear hormone receptor hepatocyte nuclear factor $4 \alpha(\mathrm{HNF} 4 \alpha)^{8}$ and the T6PNE cells used for the assay have a low level of stored fat at baseline, unlike hepatoma cell lines, which we find to be highly steatotic, making it possible to more easily study the relationship between HNF4 $\alpha$ activity and stored fat. HNF4 $\alpha$ is expressed predominantly in the liver, intestine, pancreas, and kidney, where it plays important roles in metabolic homeostasis ${ }^{12,13}$. While the traditional view of HNF4a was that the ligand binding pocket (LBP) contained a tightly bound fatty acid that was not exchangeable, we and others have shown that HNF4a undergoes ligand exchange and that receptor activity can be regulated ${ }^{7,8,14}$.

Using the insulin promoter assay, we found that fatty acids inhibit HNF4 $\alpha$ activity ${ }^{8}$, a previously undescribed activity, even though it was known that fatty acids bind in the HNF4 $\alpha$ ligand binding pocket ${ }^{15}$. Also using the insulin promoter assay, we discovered both antagonists ${ }^{8}$ and agonists ${ }^{7}$ of HNF4 $\alpha$. The HNF4 $\alpha$ activators were alverine and benfluorex, known drugs that have been used for irritable bowel syndrome and weight loss/type 2 diabetes, respectively. Of note, benfluorex has been studied in clinical trials for type 2 diabetes and proved to be effective at reducing $\mathrm{HbA1} \mathrm{c}^{16,17}$. Unfortunately, both alverine and benfluorex were relatively weak activators, making it difficult to study the role of HNF4 $\alpha$ in lipotoxic diseases. Thus, we decided to search for more potent activators.

To find more potent HNF4 $\alpha$ activators, we examined compounds that had structural similarity with alverine or benfluorex. The plant-based compounds N-trans caffeoyltyramine (NCT), and N-trans feruloyltyramine (NFT) which are associated with plant cell walls as part of a damage response ${ }^{18}$, were found to be more potent activators of the human insulin promoter-GFP transgene in T6PNE cells. Of note, NFT is derived from NCT by the action of caffeoyltyramine-O-methyltransferase ${ }^{19}$. NCT and NFT induced clearance of fat from the T6PNE cells used in the insulin promoter assay and NCT was studied in vivo, where it reversed hepatic steatosis. The mechanism by which HNF4 $\alpha$ affects hepatic fat storage is induction of lipophagy, a form of autophagy that involves fusion of lipid droplets with lysosomes and lipid hydrolysis through lysosomal acid lipase ${ }^{20}$. This is a mechanism distinct from that regulating adipocyte fat storage through hormone-sensitive lipase.

The data presented here demonstrate that HNF4 $\alpha$ meets both of the principal requirements for a molecule that can mediate the control of hepatic lipid storage. It senses fat directly by fatty acid binding to the HNF4 $\alpha$ ligand binding pocket (LBP), which controls HNF4 $\alpha$ activity. The level of HNF4 $\alpha$ activity then determines the extent of lipophagy, which releases fat from lipid vesicles in hepatocytes, thus regulating the amount of fat stored in the liver (Supplementary Fig. 1).

\section{Results}

\section{Discovery of HNF4a activators}

Previously, we screened a library of known drugs and discovered that alverine and benfluorex, which are structurally similar but used for completely distinct indications, were activators of $\mathrm{HNF} 4 \alpha^{7}$. Because alverine and benfluorex were fairly weak HNF4 $\alpha$ activators, finding stronger activators was compelling. Thus, we tested some compounds that had structural similarity with alverine and benfluorex to find additional HNF4 $\alpha$ activators (Fig. 1A). N-trans caffeoyltyramine (NCT) and N-transferuloyltyramine (NFT) were reproducibly positive hits (Fig. 1B-D). NCT was the strongest inducer of insulin promoter activity, while NFT was approximately as active as alverine. As expected for nuclear receptor ligands, which are well-known for having highly sensitive structure-activity relationships ${ }^{21}$, small structural differences resulted in large changes in activity (Fig. 1B). NCT, which was more potent than NFT, differs from NFT by a single methyl group. In plants, NCT is converted to NFT by caffeoyltyramine-O-methyltransferase ${ }^{19}$, resulting in generally higher levels of NFT than $\mathrm{NCT}^{18}$. There was no estrogenic or PPARY receptor agonist activity, both of which can produce false positives in the assay ${ }^{8,10}$ (Supplementary Fig. 2A, B)

Both NCT and NFT, and to a lesser degree N-p-coumaroyltyramine, increased INS and HNF4 $\alpha$ mRNA (Fig. 1E, F). The increase in $H N F 4 \alpha$ mRNA is particularly important, since $H N F 4 \alpha$ gene expression is the best indicator of HNF $4 \alpha$ activity in our hands, as the protein acts on its own promoter through a positive feedback loop $^{7,8,22,23}$. Both NCT and NFT exhibited doseresponsiveness in the INS promoter assay (Fig. 1D) and with respect to their ability to increase INS and $H N F 4 \alpha$ mRNA levels (Fig. 1G, H). We believe that it is likely that fatty acids and the HNF4a agonists compete for occupancy of the HNF4a LBP and that this could account for threshold effects seen in the dose-response curves.

\section{NCT and NFT act directly on HNF4a}

A prediction if the compounds act on HNF4 $\alpha$ is that $H N F 4 \alpha$ siRNA should ablate their effect. Consistent with that prediction, $H N F 4 \alpha$ siRNA repressed the effect of NCT and NFT on the INS promoter (Fig. 2A, representative images in Supplementary Fig. 3).

Binding of a compound to its target is expected to alter the structure of the target protein. This can often be detected as a change in the sensitivity to proteolytic cleavage, which is the basis for the DARTS assay ${ }^{24}$. We used this previously to determine whether compounds induce a conformational change in $\mathrm{HNF} 4 \alpha$, thus demonstrating a direct effect on the protein ${ }^{7,8}$. Consistent with our previous results, the potent HNF4 $\alpha$ antagonist BI6015 induced a conformational change in HNF4 $\alpha$ 
A

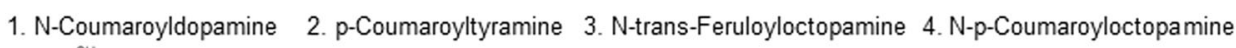
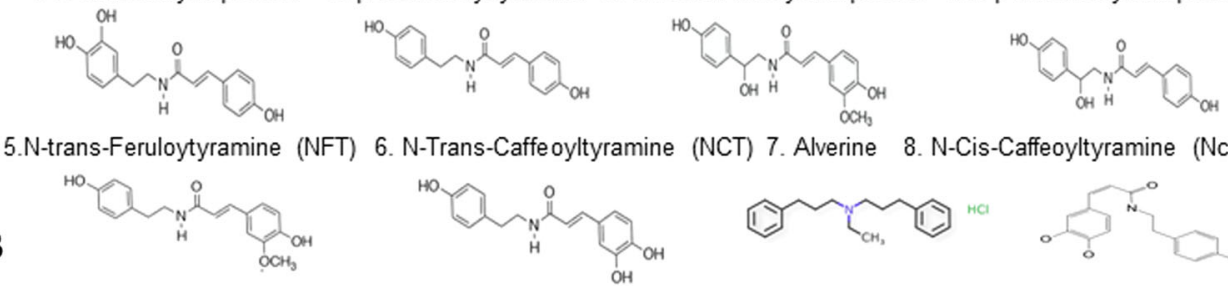

(NCT) 7. Alverine 8. N-Cis-Caffeoyltyramine (NcisCT)

B
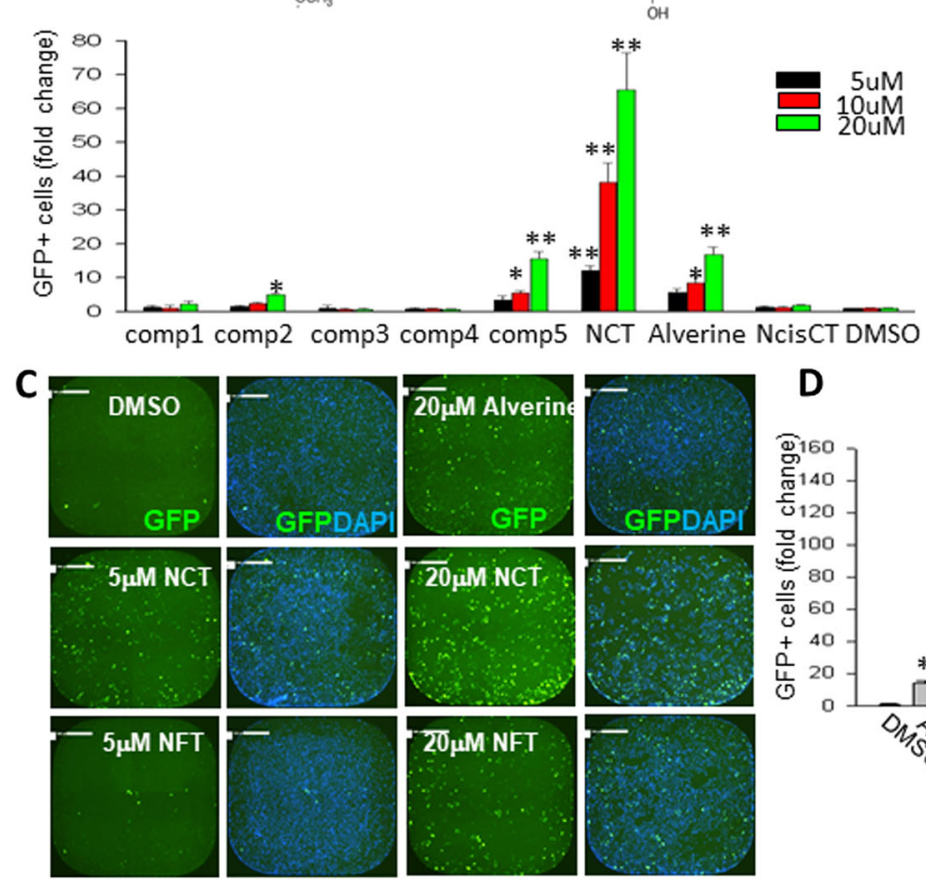

\section{D}

E

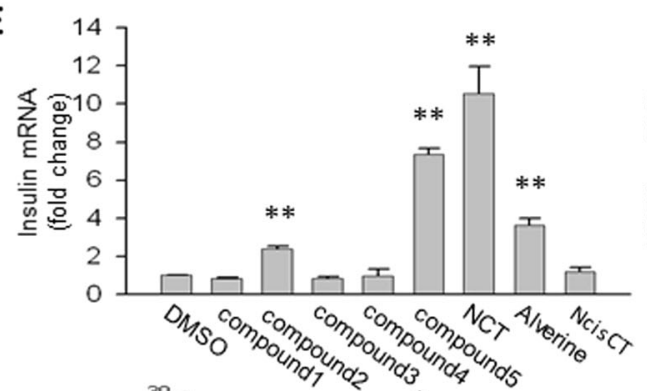

G

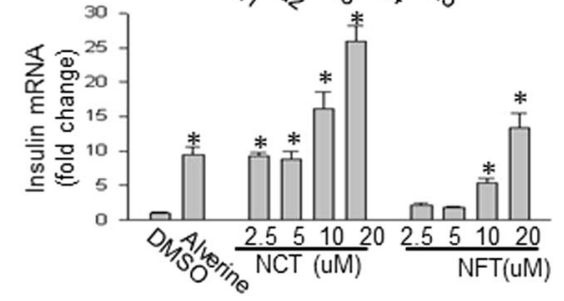

$20 \mu \mathrm{M}$ NFT

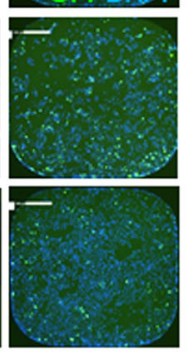

F

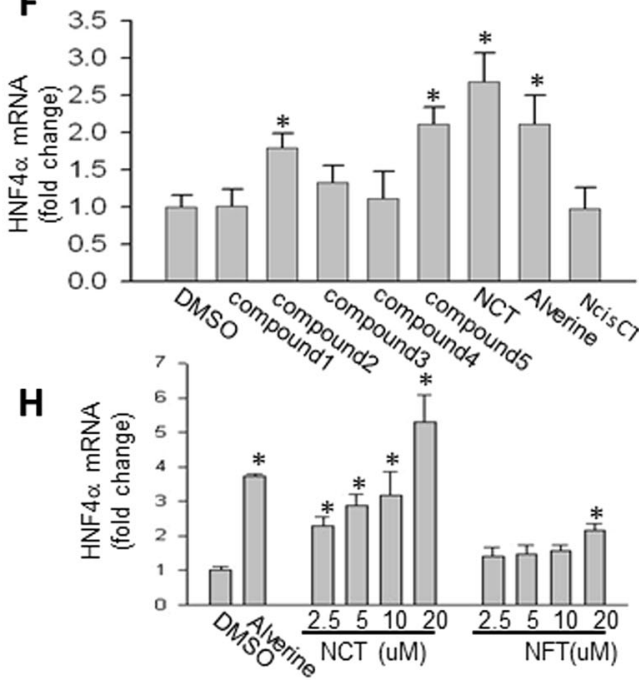

Fig. 1 Discovery of potent HNF4a agonists. A Structures of tested compounds. B Assay for HNF4a activity. Compounds from $\mathbf{A}$ were assayed as previously described ${ }^{10}$. Briefly, T6PNE cells treated with tamoxifen $(0.5 \mu \mathrm{M})$ to induce the activity of the E47 ${ }^{\mathrm{MER}}$ transgene ${ }^{10}$, were treated with the indicated compounds at 3 concentrations ( $5 \mu \mathrm{M}$, black, $10 \mu \mathrm{M}$, red, $20 \mu \mathrm{M}$, green) for 3 days, followed by fixation, staining with DAPI for nuclear visualization, and imaging for quantification of the percentage of cells expressing the insulin promoter-GFP transgene in the T6PNE cells $(N=6)$ using a Celigo imaging cytometer. $C$ Representative images of wells containing the DMSO negative control, alverine positive control, and the positive compounds NCT and NFT (green is from insulin promotor-GFP expression and blue is DAPI nuclear staining, scale bar $=500 \mu \mathrm{m}$ ). D Quantification of GFP-positive cells, reflecting activity of the human insulin promoter-GFP transgene in T6PNE cells, was done with multiple doses of NCT and NFT to demonstrate dose-responsiveness using a Celigo imaging cytometer ( $N=14)$. E, F Insulin and HNF4a mRNA levels on compounds from A were measured by qPCR as an additional measure of HNF4a activity (compound concentration was $20 \mu \mathrm{M}, N=4$ ). $\mathbf{G}, \mathbf{H} / n s u l i n$ and HNF4a mRNA levels were measured by qPCR with multiple doses of NCT and NFT $(N=4)$. Values represent the mean \pm SE. ${ }^{*} p<0.05$, ${ }^{* *} p<0.01$ (vs DMSO). 
A

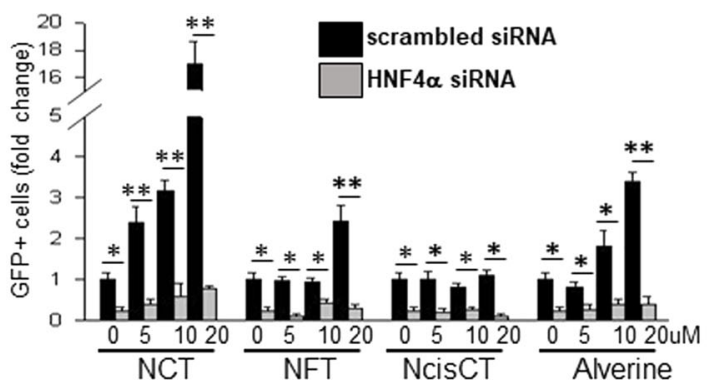

B

C
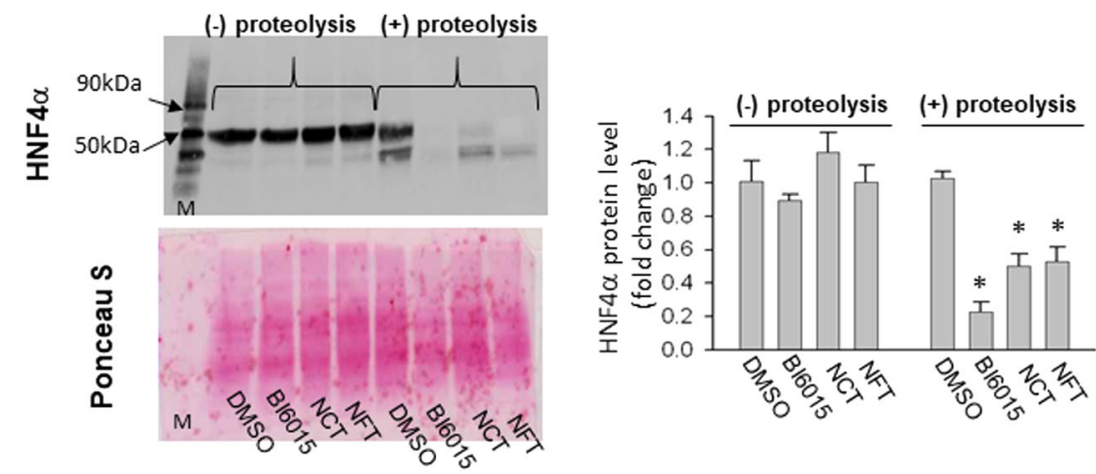

Fig. 2 NCT and NFT are HNF4a agonists. A HNF4a siRNA blocked the effect of HNF4a agonists. Scrambled or HNF4a siRNAs were transfected into T6PNE cells 2 days before compound administration. Compounds at the indicated concentrations were treated for an additional 2 days. The cells were analyzed for the percentage of cells expressing the insulin promoter-GFP transgene $(N=6)$. B DARTS assay to detect effect of compounds on HNF4a protease sensitivity. For the DARTS assay, HepG2 cells were treated with DMSO (lane 1), Bl6015 (lane 2), NCT (lane 3), or NFT (lane 4) at a concentration of 40 or $80 \mu \mathrm{M}$ for $16 \mathrm{~h}$. Total cell protein was extracted and each sample was split into two aliquots for proteolysis without (-) or with $(+)$ subtilisin and analyzed by Western blotting for HNF4a as done previously. After detection of HNF4a, the membrane was stained with Ponceau S (magenta color) as a control to ensure that the compounds did not induce nonspecific proteolysis (Lane M has MW markers). All compounds were run on the same gel. C The HNF4a level was quantified by ImageJ using the Western blots from panel $\mathbf{B}$. Values represent the mean \pm SE of 3 biological replicates, ${ }^{*} p<0.05,{ }^{* *} p<0.01$ (vs scrambled siRNA or DMSO).

(Fig. 2B, C). NCT and NFT also induced a change in HNF4 $\alpha$ proteolytic sensitivity, as expected if they act directly (Fig. 2B, C).

\section{NCT induces fat clearance from cells}

The HNF4 $\alpha$ antagonist BI6015 caused hepatic steatosis in vitro and in vivo ${ }^{8}$, and genetic deletion of $H N F 4 \alpha$ leads to hepatic steatosis ${ }^{25}$. Alverine and benfluorex were ascertained in a modification of the insulin promoter assay in which the level of insulin promoter activity was repressed with palmitate. In that modified assay, alverine and benfluorex reversed the fatty acid-mediated repression of the human insulin promoter ${ }^{7}$. Thus, it was logical to ask whether more potent HNF4 $\alpha$ agonists could alleviate steatosis. Cells were treated with $0.25 \mathrm{mM}$ palmitate for 2 days in the presence and absence of NCT or NFT $(10 \mu \mathrm{M})$. As demonstrated by Oil Red $\mathrm{O}$ and Nile Red staining, cells treated with NCT or NFT had less stored fat than control cells (Fig. 3A, quantified in B, Supplementary Fig. 2C). This was further demonstrated by quantification of the cellular triglyceride (TG) level (Fig. 3C), which produced the same result as the Nile Red staining, validating the accuracy of that assay.

\section{The sphingosine-1-phosphate transporter SPNS2 is required for fat clearance by NCT but S1P does not play a role}

Given that HNF4 $\alpha$ is a transcription factor, we believed that the mechanism by which the newly discovered HNF4 $\alpha$ agonists caused reversal of cellular steatosis would involve genes downstream of HNF4 $\alpha$. Genes with mRNA levels that were affected by both NCT and NFT and that were involved in lipid metabolism were tested for a role in fat clearance downstream of HNF4 $\alpha$ by inhibiting their expression using siRNA (siRNA validation in Supplementary Fig. 4). Of the genes tested, siRNA to only 


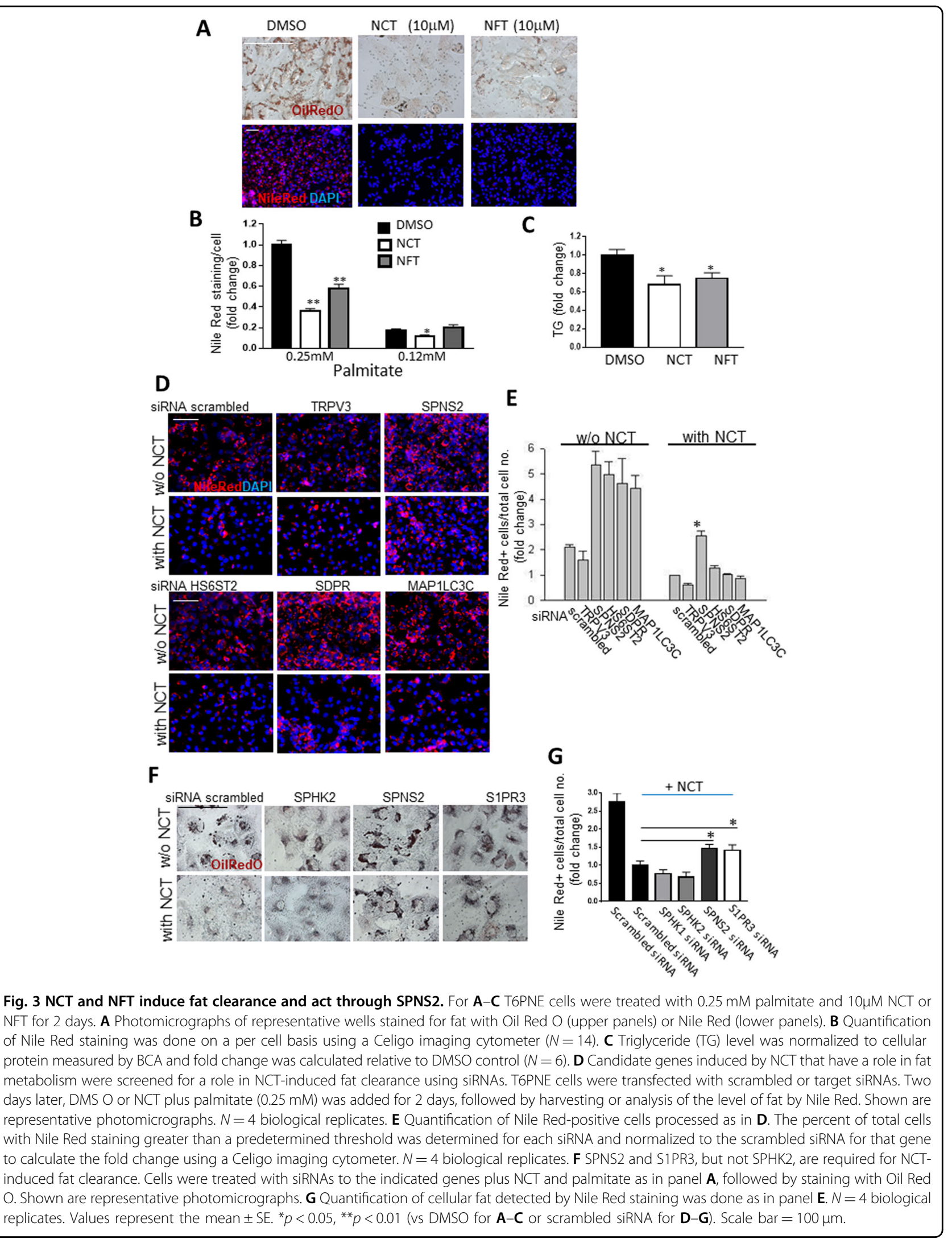


one, SPNS2, blocked the effect of NCT on fat clearance (Fig. 3D, quantified in E). SPNS2 encodes a transporter for sphingosine-1-phosphate (S1P) that moves it from the intracellular to the extracellular space. Of note, palmitate, which was used to induce steatosis and which is the major fat consumed by humans ${ }^{26}$ is a precursor for S1P synth$\mathrm{esis}^{27}$. An S1P analog that causes immunosuppression, (FTY720, aka fingolimod), has been approved for the treatment of multiple sclerosis ${ }^{28}$. S1P is synthesized from sphingosine by the action of sphingosine kinases (Sphk1, $2)^{29}$. T6PNE cells express only Sphk2 to a significant degree (GEO Accession GSE18821, GSE33432) and siRNA to Sphk2 had no effect on fat clearance induced by NCT (Fig. 3F, quantified in G) eliminating S1P as the molecule responsible for inducing fat clearance.

Once transported outside cells by SPNS2, S1P binds to a receptor in the GPCR family of signaling receptors, of which there are five family members (S1PR1-5). S1PR signaling plays an important role in diverse cell processes, but particularly in immune responses ${ }^{30}$. Only one member of the S1PR family, S1PR3, is expressed in T6PNE cells (GEO Accession GSE18821, GSE33432). S1PR3 siRNA blocked the ability of NCT to induce fat clearance (Fig. 3F, quantified in G), consistent with a model in which a molecule transported by SPNS2 that then stimulates S1PR signaling triggers fat clearance. However, this begged the question of the identity of that molecule.

\section{Dihydroceramides are required for fat clearance by NCT}

To our surprise, neither S1P nor FTY720 had any effect on fat clearance (Fig. 4A, quantified in B). However, because of the known roles of SPNS2 and S1PR3, molecules structurally related to S1P and that could be acted on by SPNS2 and S1PR3 were the obvious candidates for being the effectors in fat clearance induced by NCT. De novo S1P biosynthesis begins with palmitate and serine and proceeds through dihydrosphingosine, dihydroceramide, ceramide, and sphingosine $^{27}$ (Supplementary Fig. 1). Dihydrosphingosines have been shown to be transported by SPNS2 $^{31}$, but had no effect on fat clearance (Fig. 4A, B). In contrast, multiple dihydroceramides were highly effective at inducing fat clearance (Fig. 4A, B). This suggests that, like S1P and dihydrosphingosines, dihydroceramides are transported by SPNS2 and act through S1PRs to effect fat clearance from cells (Fig. 4C, quantified in D).

If dihydroceramides are the active molecule in fat clearance, inhibition of their conversion to ceramides by dihydroceramide desaturase 1 (DES1) should increases their level and promote fat clearance (Supplementary Fig. 1). Fenretinide is a synthetic retinoid derivative that inhibits DES1 ${ }^{32}$ but it has multiple other targets as well ${ }^{33}$. It was strongly positive in the fat clearance assay (Fig. 4A, B), as were the more specific DES1 inhibitors GT-11 and B-0027 (Fig. 4E, quantified in F) This provides further evidence that dihydroceramides are the active species responsible for the ability of NCT to induce fat clearance from cells.

\section{NCT promotes fat clearance by inducing lipophagy}

The ability of dihydroceramides to effect fat clearance from cells raised the question of the mechanism by which they act. Some studies have found a role for dihydroceramides in autophagy ${ }^{34}$. Autophagy involves alterations in LC3B through lipidation and effects on the level of the p62 autophagy receptor that can be monitored by Western blotting, with the polarity of those changes being complex and varying under different circumstances and in different cells ${ }^{35,36}$. Contrary to the classical situation in which p62 varies inversely with autophagic flux, but similar to rapamycin, which was used as a positive control to induce autophagy ${ }^{37}$, NCT induced increases in the LC3B-II to LC3B-I ratio (Fig. 5A, quantified in B), and in p62 (Fig. 5C, quantified in D).

Lipophagy is a form of autophagy that has been associated with dihydroceramides ${ }^{20}$. A central aspect of lipophagy is the cleavage of triglycerides from lipid droplets by lysosomal acid lipase $(\mathrm{LAL})^{20}$. Direct association between lipid droplets and lysosomes has been demonstrated $^{38}$. To determine whether lipophagy plays a role in fat clearance induced by NCT, we used the LAL inhibitor Lalistat $2^{39}$. Consistent with NCT acting by stimulation of lipophagy, Lalistat 2 inhibited the ability of NCT to promote fat clearance (Fig. 5E, quantified in F, Supplementary Fig. 5).

\section{NCT reverses hepatic steatosis}

Having established that NCT reduces the level of stored fat in cells in vitro, it was of interest to determine its effect in vivo. We focused on the liver, the organ expressing the highest level of HNF4 $\alpha$ and a major site of pathological fat storage, i.e., NAFLD. HNF $4 \alpha$ has been recognized as playing an important role in NAFLD ${ }^{40}$. Adipose cells, the other major site of fat storage, do not express HNF4 $\alpha$. NCT was administered by IP injection ( $200 \mathrm{mg} / \mathrm{kg}$ bid) for two weeks to C57BL/6 J DIO mice maintained on a $60 \%$ fat calorie diet. The dose was selected on the basis of a doseresponse study in which mice were injected with $\mathrm{NCT}$ at increasing doses (30, 60, 120 and $240 \mathrm{mg} / \mathrm{kg}$ bid for 3 days), which was well tolerated (Supplementary Table 1).

After two weeks, the livers of the NCT-injected mice exhibited a shift in the color of the liver from yellow to red, as expected with a decrease in fat content (Fig. 6A, B). Livers from NCT-injected mice weighed less than those of the control mice, as expected if NCT was stimulating loss of hepatic fat (Fig. 6C) and this was borne out by a reduction in the hepatic triglyceride content (Fig. 6F). Analysis of liver sections revealed a decrease in stored fat by Oil Red O staining (Fig. 6I, J). Control and NCT-injected 
A

DMSO

NCT

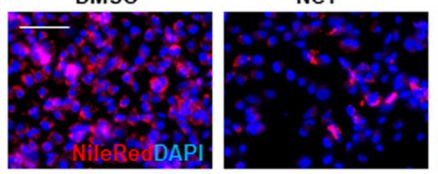

Fenretinide

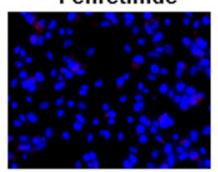

S1P
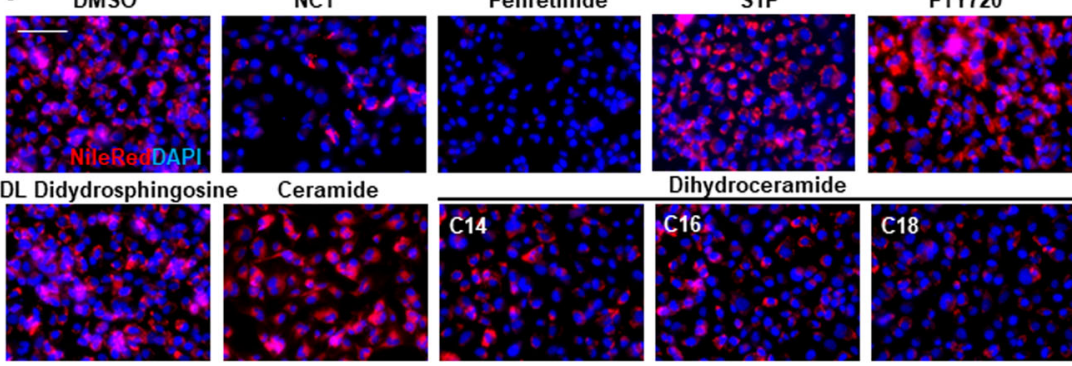

Dihydroceramide
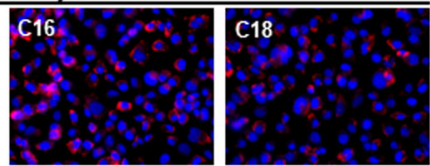

B

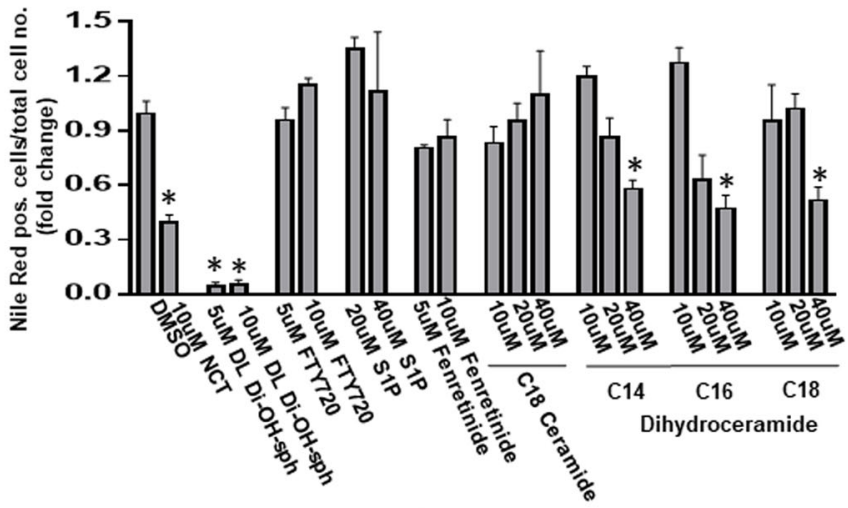

C

E
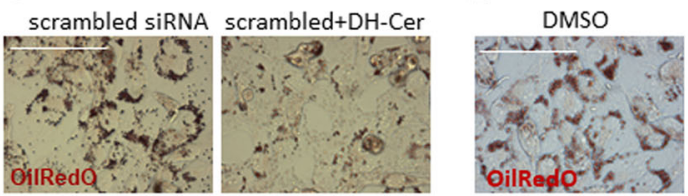

NCT $(15 \mu \mathrm{M}) \quad$ Fenretinide $(5 \mu \mathrm{M})$

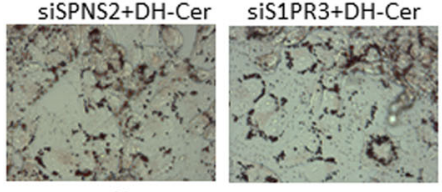

siS1PR3+DH-Cer
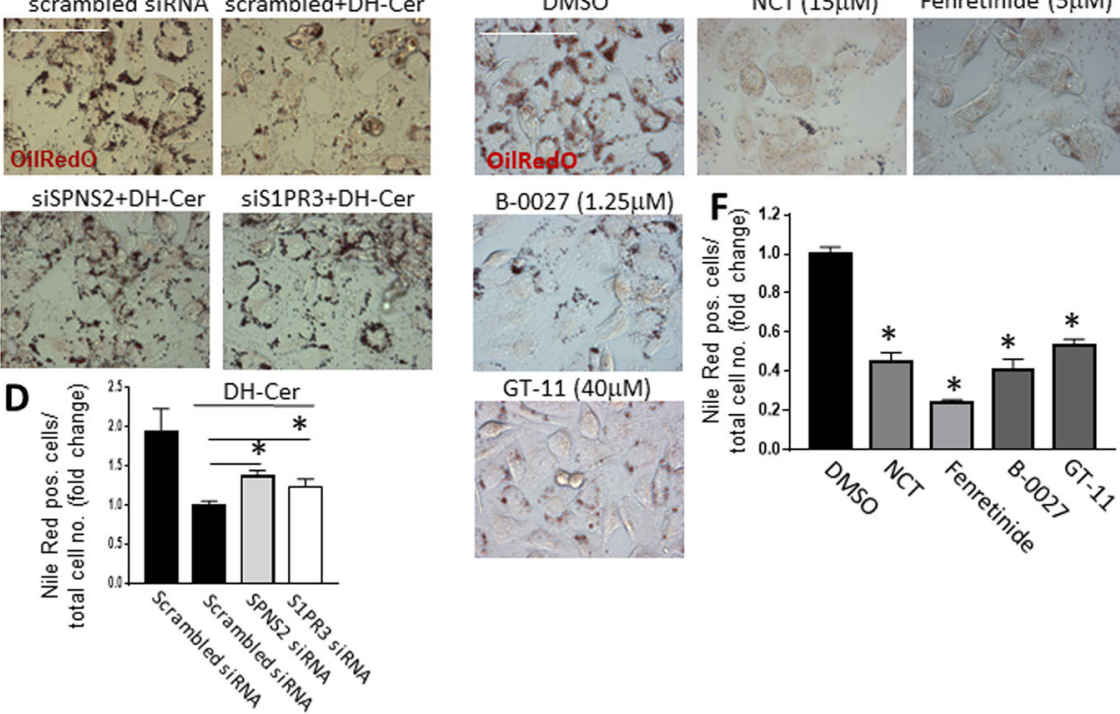

Fig. 4 Dihydroceramides (DH-Cer) induce fat clearance. A Representative photomicrographs of T6PNE cells treated for 2 days with $0.25 \mathrm{mM}$ palmitate and the indicated compounds, followed by staining for fat with Nile Red. B Quantification of Nile Red staining from A. C Inhibition by siRNA to SPNS2 or S1PR3 of fat clearance induced by DH-Cer. T6PNE cells were transfected with siRNAs to SPNS2 or S1PR3. Two days later, DH-Cer was added for 2 days, followed by staining with Oil Red O. D Quantification of the number of cells positive for Nile red from $\mathbf{C}$, demonstrating that DHCer-induced fat clearance requires SPNS2 and S1PR3. E DES-1 inhibitors GT-11and B-0027 increase fat clearance. T6PNE cells were treated with $0.25 \mathrm{mM}$ palmitate and DMSO or NCT. F Quantification of the Nile Red cells shown in $\mathbf{E}$. Values represent the mean \pm SE of 6 biological replicates, ${ }^{*} p<0.05$ (vs DMSO or scrambled siRNA). Scale bar $=100 \mu \mathrm{m}$.

mice gained weight to a similar degree and both groups remained healthy and active (Supplementary Fig. 6).

The lack of a difference in body weight combined with the decreased liver weight implied that fat released from the liver must be present elsewhere. Consistent with that, there was an increase in epididymal fat pad mass (Fig. 6D, E). The shift of fat from the liver to adipose tissue suggested that fatty acids had to have traveled through the circulation to shift from one tissue to another. This would be expected if lipophagy was being induced by NCT, as lipophagy involves 

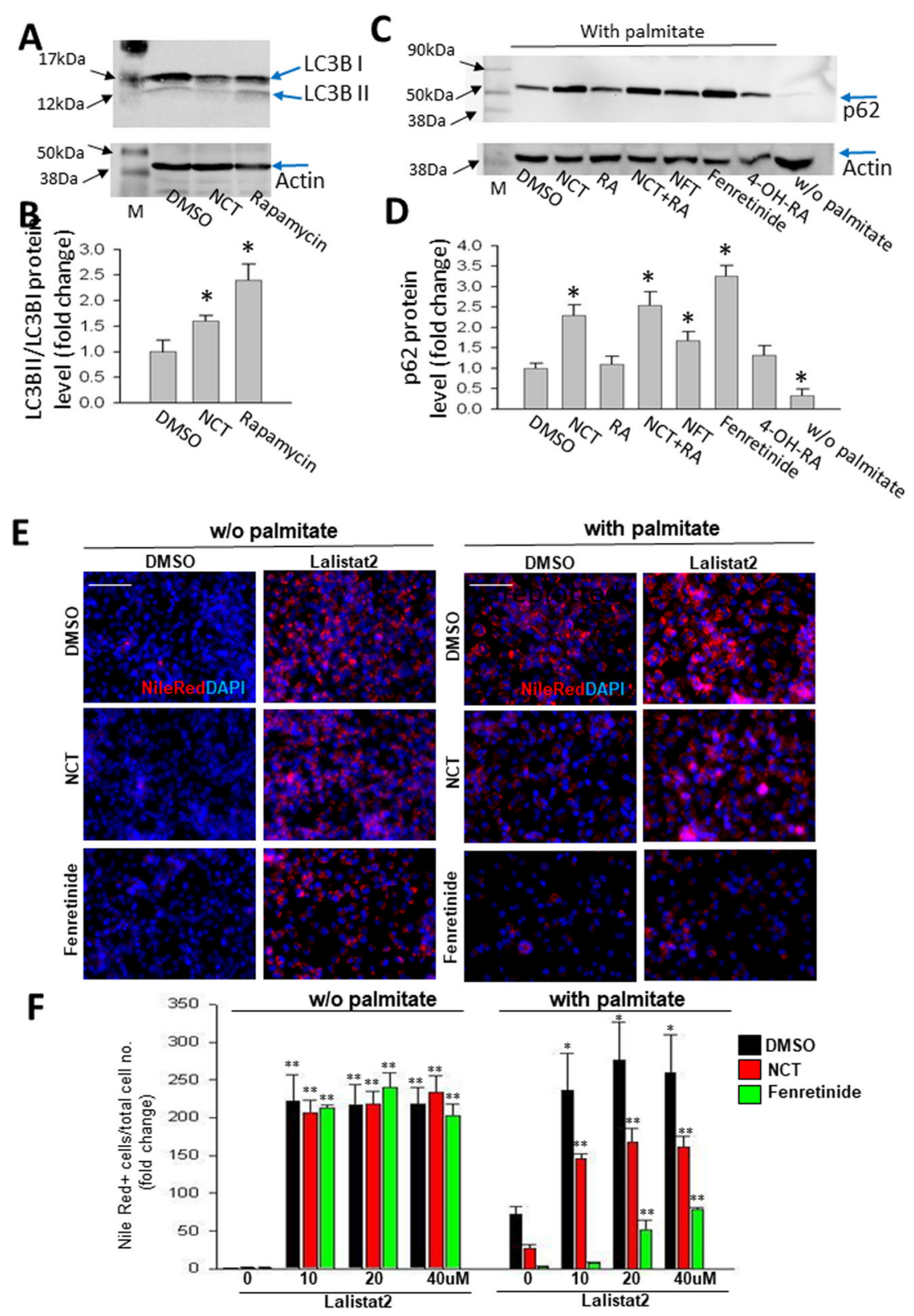

Fig. 5 NCT promotes fat clearance by inducing lipophagy. T6PNE cells were treated with $0.25 \mathrm{mM}$ palmitate for 2 days with the indicated compounds, followed by harvesting for Western blot and imaging. A Anti-LC3B Western blot demonstrating an increased ratio of LC3B II to LC3B I. For

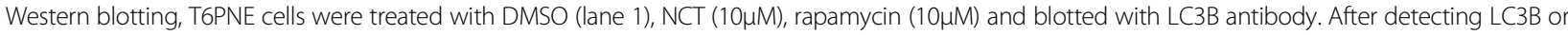
p62 (panels $\mathbf{A}, \mathbf{C}$ ), the same membrane was reblotted with anti- $\beta$-actin antibody to ensure equal amounts of protein in each lane. $\mathbf{B}$ Quantification of

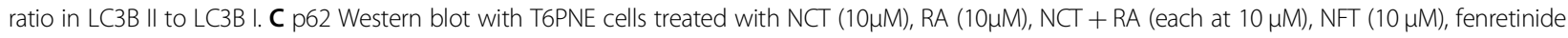
$(5 \mu \mathrm{M}), 4-\mathrm{OH}-\mathrm{RA}(20 \mu \mathrm{M})$, or without palmitate. D Quantification of p62 protein expression normalized to actin. The same membrane was reblotted with anti-ß-actin antibody to ensure equal amounts of protein in each lane. Fenretinide had a statistically significant effect but retinoic acid did not. E Lysosomal acid lipase is required for the effect of NCT on fat clearance. Representative images of T6PNE cells treated for 2 days with or without palmitate, NCT $(5 \mu \mathrm{M})$, fenretinide $(5 \mu \mathrm{M})$ and the LAL inhibitor Lalistat2 $(20 \mu \mathrm{M})$, followed by staining with Nile Red to visualize intracellular fat. F Quantification of the conditions shown in panel E. Values represent the mean \pm SE of 3-4 biological replicates. All fold changes were calculated relative to $0 \mu \mathrm{M}$ Lalistat2 in the absence of palmitate, ${ }^{*} p<0.05,{ }^{* *} p<0.01$ (DMSO vs. NCT or each NCT vs NCT + Lalistat2). Scale bar $=100 \mu \mathrm{m}$.

the release of fatty acids from cells through the action of LAL, which should lead to an increase in circulating free fatty acids (FFA). To test that prediction, we employed a protocol used in humans ${ }^{41}$ and mice ${ }^{42}$ in which a glucose challenge is used to stimulate insulin secretion, which inhibits FFA release from adipocytes, leaving liver-derived 


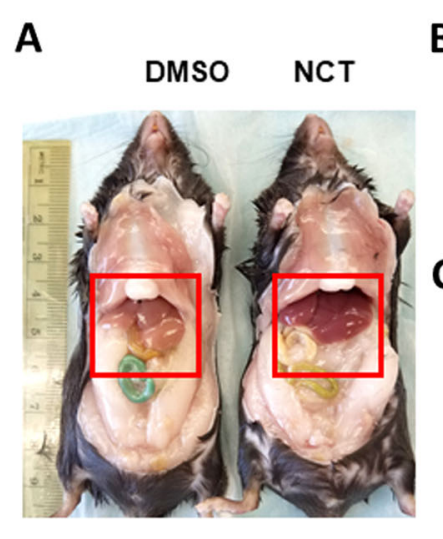

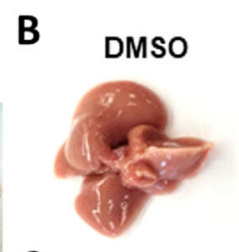

C

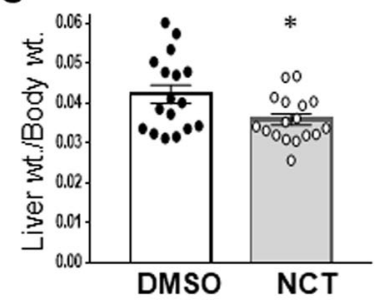

D
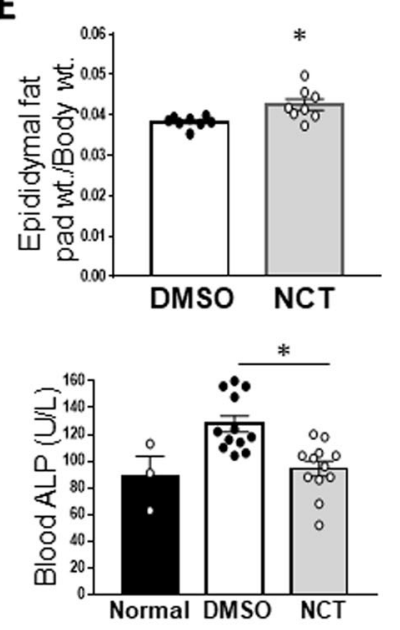

DMSO

NCT

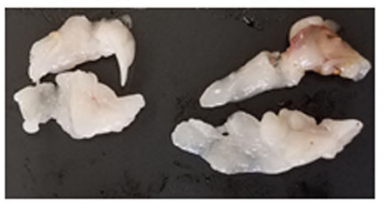

F

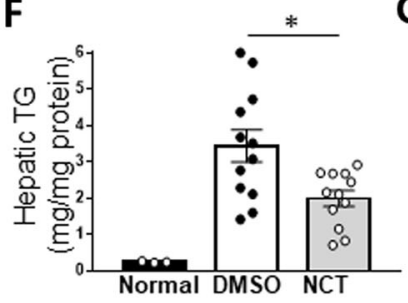

H

G

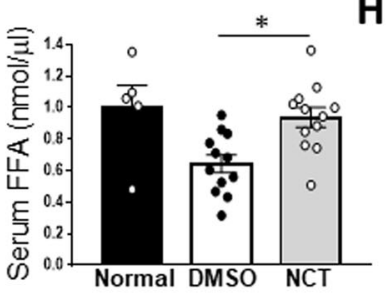

I

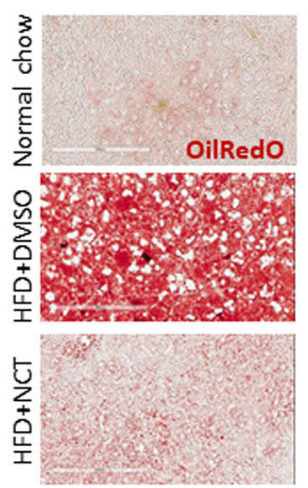

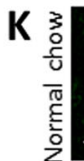
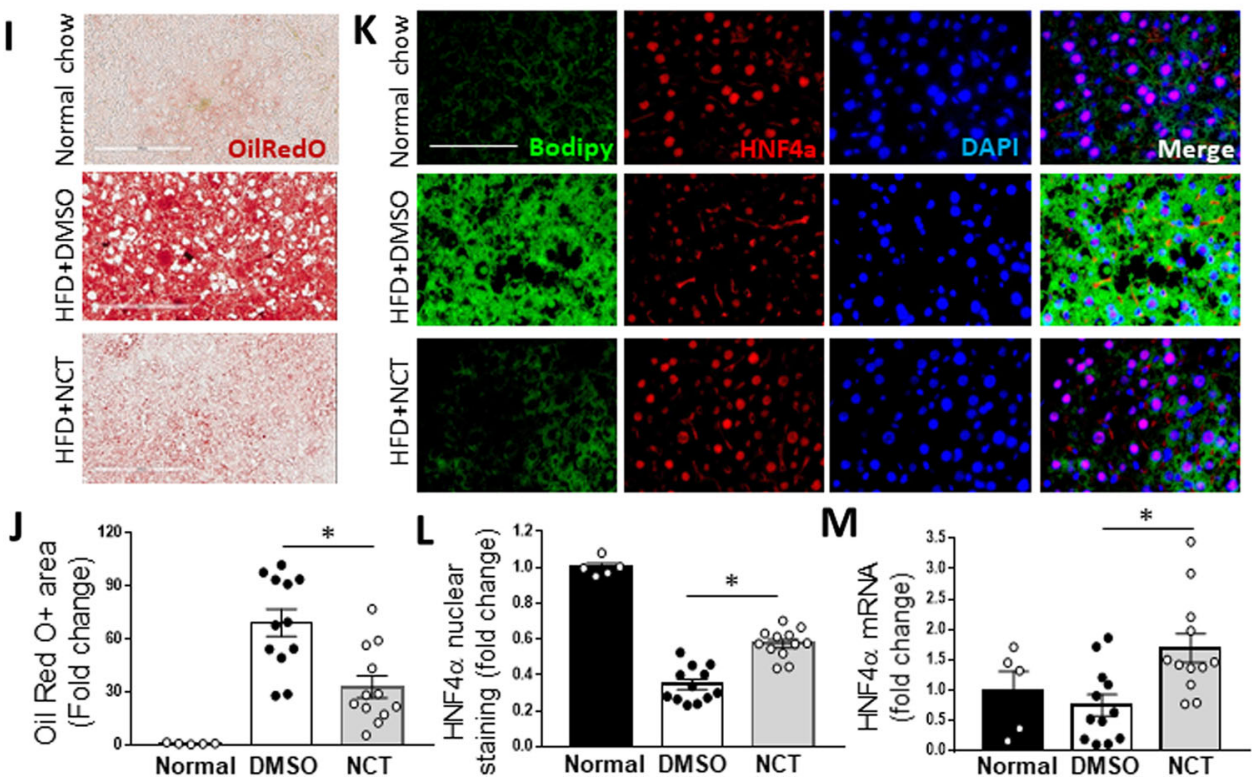

Fig. 6 NCT reverses hepatic steatosis in vivo. DIO mice (C57BL/6 J) were injected intraperitoneally with NCT (200 mg/kg bid) for two weeks, followed by harvesting of organs. A Red box indicates the area of the liver, demonstrating a marked difference in color. B Dissected liver from representative mice indicating difference in color and weight (quantified in $\mathbf{C}, N=12$ for each group). $\mathbf{D}$ Epididymal fat pads from representative mice showing increased weight with NCT (quantified in $\mathbf{E}, \mathrm{N}=12$ for each group). $\mathbf{F}$ Hepatic triglyceride (TG) content normalized to hepatic protein (Normal chow control, $N=3$, DMSO and NCT, $N=12$ ). $\mathbf{G}$ Serum free fatty acid (FFA) level (Normal chow control, $N=5$ and DMSO and $N C T, N=12$ ). H Blood alkaline phosphatase (ALP) level (Normal chow control, $N=3$ and DMSO and NCT, $N=12$ ). I Representative photomicrograph of hepatic Oil Red $\mathrm{O}$ staining (scale bar $=200 \mu \mathrm{m}$ ). J Oil Red O quantification. The percent of the liver section positive for Oil Red O was measured using Image $\mathrm{J}$ with a consistent threshold setting and normalized to liver sections from mice fed normal chow. K Representative liver sections stained for Bodipy (green), HNF4a (red), DAPI (blue) and merged images in mice fed normal chow or HFD plus DMSO or NCT. L Quantification of HNF4a nuclear staining. HNF4a nuclear staining intensity with non-specific cytoplasmic staining from same cell subtracted. Normalized to livers from mice fed normal chow. M Quantification of hepatic HNF4a mRNA level. Dots indicate individual mice. Values represent the mean \pm SE, Normal chow control, $N=3-5$; DMSO and NCT, $N=12 .{ }^{*} p<0.05,{ }^{* *} p<0.01$. Scale bar $=100 \mu \mathrm{m}$. 
FFA as the major source of circulating FFA. Consistent with our hypothesis, NCT induced an increase in free fatty acids (Fig. 6G). Serum TG was increased by NCT at week 1 of NCT administration but not at week 2, the end of the study (Supplementary Fig. 7A).

\section{ALP was decreased by NCT}

Markers of liver injury are elevated in NAFLD, including alkaline phosphatase (ALP) ALP has been studied as an early indicator of the transition to hepatic fibrosis as part of the progression from NAFLD to non-alcoholic steatotic hepatitis $(\mathrm{NASH})^{43,44}$. Mice treated with NCT exhibited decreased ALP (Fig. 6H). There was no change in the level of other markers in the VetScan panel ${ }^{45}$ (Supplementary Fig. 7B).

\section{NCT reverses negative effects of fatty acids on HNF4a expression}

HNF $4 \alpha$ feeds back on its own promoter in a positive feedback loop ${ }^{23,46,47}$ and is the best marker of HNF4 $\alpha$ activity in our experience ${ }^{7,8}$. In vitro, NCT and NFT induced HNF4 $\alpha$ expression in T6PNE cells (Fig. 1G) and primary human hepatocytes (Supplementary Fig. 8). In vivo, we showed previously that the potent $H N F 4 \alpha$ antagonist BI6015 caused loss of HNF4 $\alpha$ expression in the liver $^{8}$, so we tested whether NCT had an effect in vivo. In control DIO mice, HNF4 $\alpha$ protein was decreased compared with mice on a normal chow diet, as expected given our previous finding that fatty acids inhibit $H N F 4 \alpha$ activity $^{8}$ (Fig. 6K, L). Interestingly, we did not detect a decrease in HNF4a mRNA with HFD (Fig. 6M), which could be due to post-translational regulation of $\mathrm{HNF}_{4} \alpha^{48}$. NCT reversed the loss of HNF4 $\alpha$ protein (Fig. 6K, L) and mRNA (Fig. 6M).

\section{CYP26A1, an HNF4a downstream target, plays an important role in the induction of fat clearance}

In the mouse pancreas and T6PNE cells, NCT induced SPNS2 expression (Supplementary Figure 9), which is required for the reversal of cellular steatosis (Fig. 3). However, NCT did not induce a change in SPNS2 expression in the mouse liver (Supplementary Fig. 9) or primary cultured human hepatocytes (Supplementary Fig. 8). Because we deemed it unlikely that NCT was acting by a fundamentally different mechanism to clear fat from the liver than in T6PNE cells in vitro which was originated from human pancreas, we examined for other genes induced by NCT in the liver that might be involved in the same pathway (GSE172234, Supplementary Fig. 10). CYP26A1 is induced by $H N F 4 \alpha$ in conjunction with retinoic acid (RA) ${ }^{49}$ and converts RA to multiple metabolites, including 4-oxo-RA, 5,6 epoxy$\mathrm{RA}$, and 4-OH-RA ${ }^{50}$. RA is synthesized in the liver and so is abundant there ${ }^{51}$.
As we had shown previously that fenretinide, a synthetic retinoid that inhibits DES1, induced fat clearance from T6PNE cells, we hypothesized that CYP26A1 was a good candidate for playing a role in NCT-induced fat clearance from the liver. NCT induced CYP26a1 in the mouse liver (Fig. 7A), cultured human hepatocytes (Supplementary Fig. 8) and in T6PNE cells (Fig. 7B). The generalized CYP inhibitor $\mathrm{ABT}^{52}$ and the specific CYP26 inhibitor talarazole $^{53}$ inhibited fat clearance by NCT (Fig. 7C, D).

Having demonstrated a role for CYP26A1 in fat clearance downstream of NCT, we hypothesized that one of the retinoic acid metabolites produced by CYP26 should induce fat clearance. Consistent with that, 4-OH RA induced fat clearance from T6PNE cells (Fig. 7E, F). Interestingly, 4-OH-RA also induced an increase in CYP26A1 mRNA (Fig. 7B) Thus, RA and its metabolites play important roles in fat storage in the liver through transcriptional and post-transcriptional mechanisms, respectively (Supplementary Fig. 1).

\section{NCT induces dihydroceramide production}

A key prediction of our model of the control of hepatic fat storage by HNF4 $\alpha$ is that HNF4 $\alpha$ should increase the production of dihydroceramides (Supplementary Fig. 1). This was tested in vitro and in vivo using a lipidomic approach $^{54}$. Lipidomic analysis revealed that multiple dihydroceramides were increased by NCT in T6PNE cells. Strikingly, there was a strong correlation between the dihydroceramides produced in response to NCT and those induced by fenretinide (Fig. $8 \mathrm{~A}, R^{2}=0.79$ ), consistent with the model (Supplementary Fig. 1) that a downstream effect of NCT is to inhibit DES1. Both NCT + RA (used to induce CYP26A) and fenretinide induced a substantial decrease in the ratio of ceramide produced by the action of DES1 relative to the corresponding dihydroceramide (Fig. 8B, Supplementary Tables 2 and 4). This was evident as well in lipidomic analysis of livers from mice injected IP with NCT (Fig. 8C, Supplementary Tables 3 and 5). This is consistent with NCT administration in vivo resulting in inhibition of DES1 and consequent increased dihydroceramide production in the liver as predicted by our model (Supplementary Fig. 1).

\section{Discussion}

Understanding the regulation of fat storage in the liver is vital to developing treatments for fatty liver disease, a major public health problem ${ }^{3}$. Here, we report the discovery of a previously unknown pathway by which HNF4 $\alpha$ controls hepatic fat storage. That discovery was made possible by the discovery of potent HNF4 $\alpha$ agonists, also reported here. Stimulation of HNF4 $\alpha$ activity with the HNF4 $\alpha$ agonist NCT led to reversal of hepatic steatosis in the short time frame of two weeks. Encouragingly for its therapeutic potential, NCT treatment was completely 
A

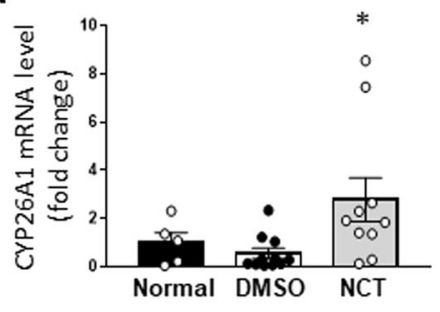

C
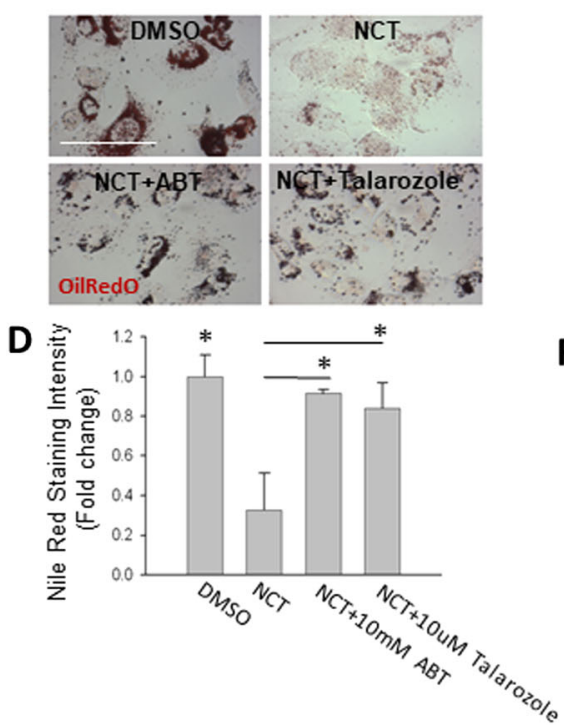

B

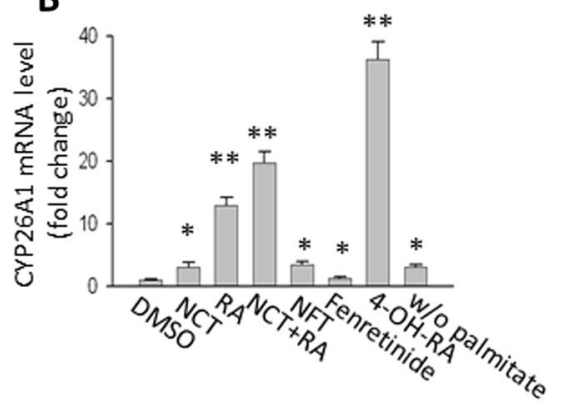

E
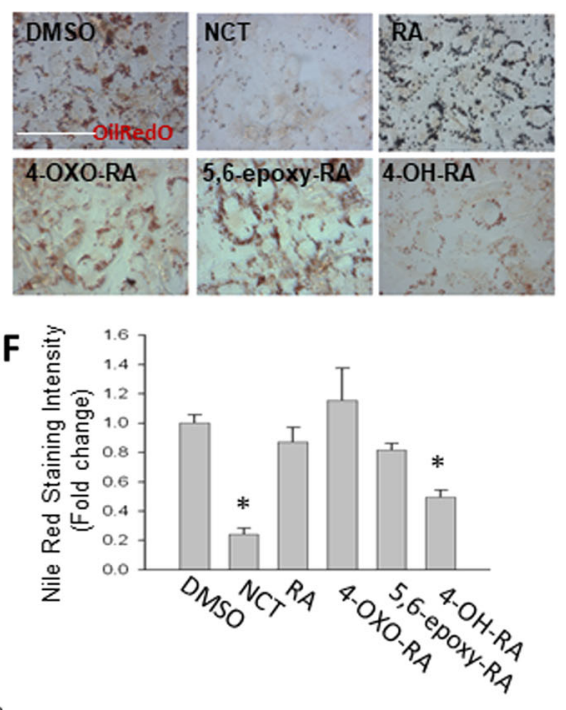

Fig. 7 Fat clearance by NCT acts through CYP26A1. A RT-PCR analysis of hepatic CYP26A1 mRNA level (Normal chow control; $N=5$ and for DMSO and $N C T ; N=10)$. B RT-PCR analysis of CYP26A1 mRNA level in T6PNE cells treated for 2 days with DMSO, NCT $(10 \mu M), R A(10 \mu M), N C T+R A(10 \mu M)$, NFT $(20 \mu \mathrm{M})$, fenretinide $(5 \mu \mathrm{M}), 4-\mathrm{OH}-\mathrm{RA}(20 \mu \mathrm{M})$ on $0.25 \mathrm{mM}$ palmitate and DMSO w/o palmitate. C CYP26A1 is required for fat clearance by NCT. T6PNE cells treated with palmitate $(0.25 \mathrm{mM})$ plus the indicated compounds for 2 days, including the inhibitors ABT (10 mM, broad CYP inhibitor) and Talarozole (10uM, selective CYP26 inhibitor). D Quantification of the effect of CYP inhibitors on fat clearance by NCT (vs NCT for significance). E Metabolites from CYP26-mediated RA metabolism induce fat clearance. Representative images of T6PNE cells treated for 2 days with NCT or the RA

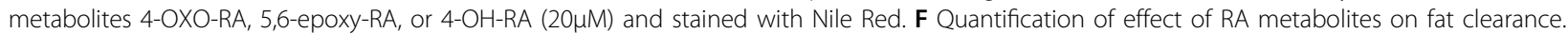
Values represent the mean \pm SE of 4 biological replicates, ${ }^{*} p<0.05,{ }^{* *} p<0.01$ (vs DMSO). Scale bar $=100 \mu \mathrm{m}$.

nontoxic and led to a decrease in ALP, an important marker of liver injury and progression from NAFLD to NASH. ALP has been put forth as a marker of hepatic fibrosis $^{43}$ and is also used commonly as a biomarker for biliary cholangitis ${ }^{55,56}$.

The HNF4 $\alpha$ agonists described here are structurally similar to alverine and benfluorex, known drugs that we found previously to be HNF4 $\alpha$ activators ${ }^{7}$. The strategy of drug repurposing that we began with is being used for many diseases, including COVID-19 $9^{57}$, but there are few examples of success ${ }^{58}$. Alverine and benfluorex are weak HNF $4 \alpha$ activators and are unsuitable for in vivo use, but they served as an important starting point for the efforts described here, and thus can be taken as partial validation of the strategy. However, NCT and NFT are much more potent, making possible in vivo and mechanistic studies. NCT and NFT are found in plants, including some consumed by humans. They have no known role as nuclear receptor ligands or mediators of plant metabolism, and there is no HNF4a homolog in plants. There have been many reports of compounds found in plants and plant extracts that have beneficial effects on disorders caused by fat excess, including type 2 diabetes and fatty liver disease ${ }^{59,60}$.

NCT and NFT are found in association with plant cell walls. They are induced in response to damage and are thought to play a role in pathogen defense ${ }^{18}$. However, little is known about their function. In animal cells and in mice, they have been shown to have anti-inflammatory properties $^{61}$. The concentration of NCT in most plants is 

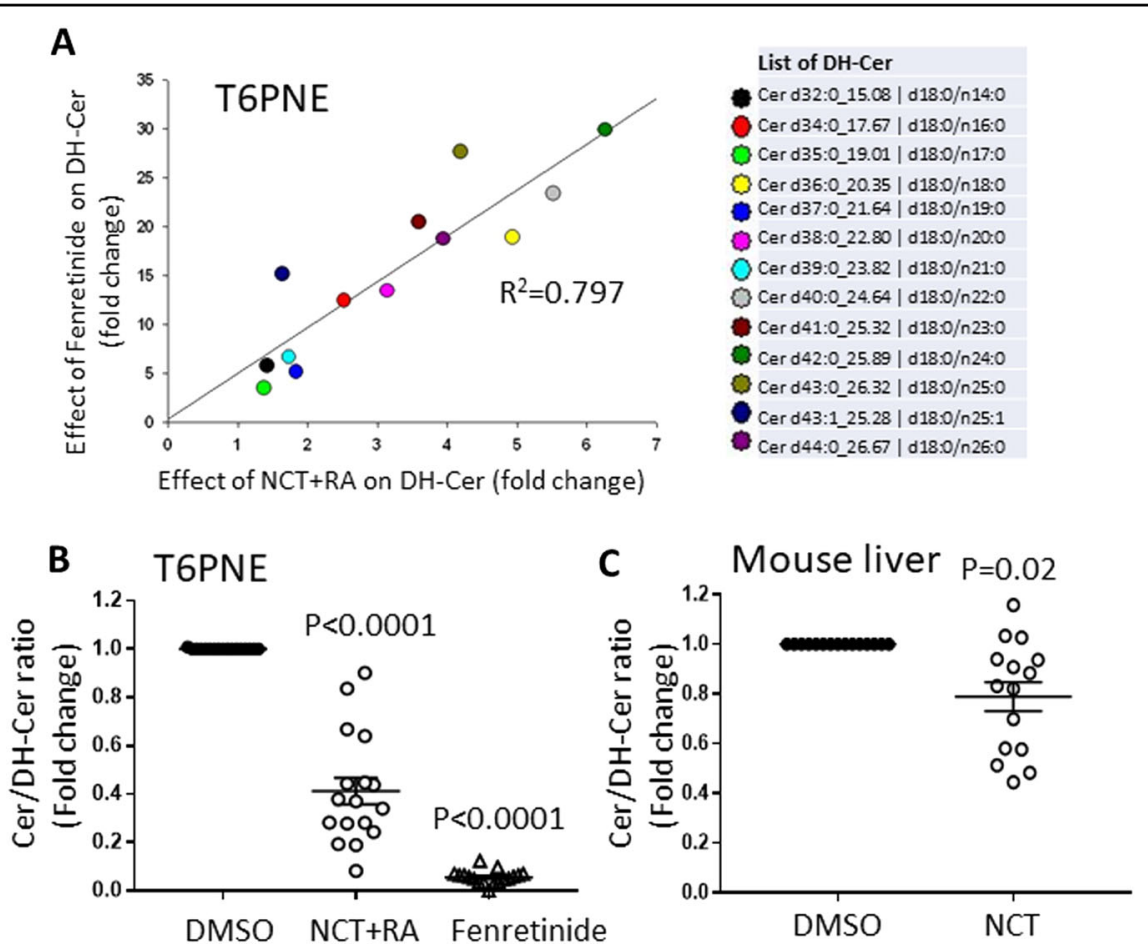

Fig. 8 Dihydroceramides were increased in T6PNE cells and mouse liver by NCT. Ceramides and dihydroceramides were measured in vitro and in vivo following treatment with the indicated compounds. A T6PNE cells were harvested 2 days after treatment with DMSO, NCT + RA (10 MM) and Fenretinide $(5 \mu \mathrm{M})$. NCT and fenretinide induced multiple identical dihydroceramides $\left(R^{2}=0.797\right)$. B The ceramide $(C e r) /$ dihydroceramide $(\mathrm{DH}-\mathrm{Cer})$ ratio was decreased in T6PNE cells treated with NCT + RA or Fenretinide. C Livers from mice treated with DMSO or NCT for 2 weeks. The ceramide/ dihydroceramide ratio decreased in response to NCT ( $P=0.02$, see Supplementary Tables 2 and 3 for individual ratios and raw data). $N=3-4$ biological replicates (vs DMSO). Note that C17 fatty acids are derived from consumption of plants and are not produced in mammals.

low, as it is an NFT precursor, being converted to NFT by O-methylation of the 3-hydroxyl group of the phenylpropenic acid moiety ${ }^{18}$. NFT is more abundant, being found in various plants at a few tens of micrograms per gm of dry plant, but that will vary depending on the degree to which the compounds were induced prior to harvest ${ }^{62}$. Given their poor oral bioavailability and low abundance in plants that are commonly consumed as food, NCT and NFT are unlikely to be physiologically relevant sources of $\mathrm{HNF} 4 \alpha$ ligands in most human diets, but that is a question worthy of additional investigation.

The mechanism that we found for the control of hepatic fat storage by HNF4 $\alpha$ involves the induction of lipophagy, a form of autophagy ${ }^{20}$. Genetic knockout studies and molecules that stimulate autophagy have implicated autophagy in fatty liver disease ${ }^{63}$. However, the induction of lipophagy by HNF4 $\alpha$ has not been suspected previously. An advantage of stimulating lipophagy by $H N F 4 \alpha$ agonists rather than by general stimulators of autophagy ${ }^{64}$ is that HNF4 $\alpha$ expression is highly tissue restricted, being expressed predominantly in the liver, pancreas, kidney and intestine. We did not observe any systemic effects of $\mathrm{NCT}$, and in fact were unable to establish a maximum tolerated dose because of the lack of toxicity. The fat released from the liver appeared to be taken up by adipocytes, which do not express HNF4 $\alpha$.

$\mathrm{HNF} 4 \alpha$ is a nuclear receptor transcription factor, so we hypothesized that the mechanism by which it stimulates lipophagy must involve HNF $4 \alpha$-mediated transcriptional effects on genes that ultimately promote lipophagy. In the T6PNE cells used in the insulin promoter assay, upregulation by NCT of SPNS2, a transporter, led to the identification of dihydroceramides as playing a key role in stimulating lipophagy downstream of HNF4 $\alpha$. In the liver in vivo, HNF4 $\alpha$ acts in conjunction with retinoic acid to activate CYP26A1 transcription. In one of the many feedback loops in this system, RA is then metabolized by CYP26 itself to a number of metabolites ${ }^{65}$, of which we found at least one, 4-OH RA, to inhibit the dihydroceramide metabolizing enzyme DES1 to increase the production of dihydroceramides. 4-OH RA also induces CYP26A expression. Complex and interacting feedback loops appear to be a central feature of the pathway described here. One of the most important of those is the inhibition by palmitate of HNF4 $\alpha$ activity, which should lead to decreased lipophagy and consequent increased fat storage. However, de novo dihydroceramide synthesis begins with palmitate, the major fat consumed by humans 
and a major effector of lipotoxicity ${ }^{26}$. We show here that dihydroceramides stimulate lipophagy, resulting in decreased fat storage, opposing its negative effect on $\mathrm{HNF} 4 \alpha$ as a direct inhibitor through binding to HNF4 $\alpha^{8}$ (Supplementary Fig. 1).

Dihydroceramides have been implicated in lipotoxic diseases, including hepatic steatosis and type 2 diabetes ${ }^{34}$. They have been measured in the blood type 2 diabetes and cardiovascular disease ${ }^{66}$. Genetic ablation of DES1 improves insulin resistance and hepatic steatosis ${ }^{67}$, but dihydroceramide function in those diseases has not been well understood. Under our model, the highest concentration of secreted dihydroceramides will be in the immediate vicinity of their site of export by SPNS2, so it is likely that they act primarily in an autocrine and/or paracrine manner through S1PRs on nearby cells. We found that S1PR3 is necessary for the action of NCT and dihydroceramides in T6PNE cells and so must be a receptor for dihydroceramides, but the other four receptors have not been studied in this regard. They are expressed in complex patterns, which could potentially contribute to effects in other tissues. For example, dihydroceramides play important roles in hematopoietic stem cells $^{68}$, so restricting activity primarily to the organs that are affected by a particular disease such as NAFLD by targeting HNF4 $\alpha$ could potentially avoid undesirable side effects. An interesting area for future studies will be to determine how S1P receptors signal to lipid vesicles to promote lipophagy.

The model described above does not require the existence of an endogenous HNF4 $\alpha$ agonist, and no such agonist has been found. Rather, HNF4 $\alpha$ appears to exhibit a high level of basal activity in the absence of ligand binding, with fatty acids acting as endogenous antagonists ${ }^{14}$. The high potency of NCT as an HNF4 $\alpha$ agonist allows for activation of lipophagy even in the face of a high, pathological level of endogenous fat. The finding that fatty acids regulate HNF4 $\alpha$ activity combined with the finding that HNF4 $\alpha$ regulates lipophagy support a model in which HNF4 $\alpha$ is repressed by high levels of ingested fat under fed conditions. This leads to suppression of hepatic lipophagy and storage of any available fat. Under the condition of starvation, HNF4 $\alpha$ will not have bound fat, as found for linoleic acid ${ }^{14}$, and thus will be more active, leading to increased hepatic lipophagy and release of stored fat. Ingestion of a high level of dietary fat will lead to constitutive downregulation of HNF4 $\alpha$ activity and thus to hepatic steatosis.

In addition to hepatic steatosis, HNF4 $\alpha$ is implicated in a number of other diseases ${ }^{69}$, that affect tissues with high HNF $4 \alpha$ expression, including type 2 diabetes, where HNF4 $\alpha$ has been found to be a type 2 diabetes gene in a number of GWAS studies ${ }^{70}$. Haploinsufficiency for HNF4 $\alpha$ causes MODY1, a monogenic form of diabetes ${ }^{71}$.
Thus, HNF4a agonists could be of benefit in other settings in addition to NAFLD. Of note, hepatic steatosis and consequent hepatic insulin resistance play an important role in T2D pathogenesis, so ameliorating NAFLD could have beneficial effects on diabetes independent of any effects on the islet ${ }^{72}$. In the intestine, HNF4 $\alpha$ has been implicated in inflammatory bowel disease by GWAS studies $^{73}$. A site of significant HNF4 $\alpha$ expression is the kidney, and obesity is a strong risk factor for the development of renal disease ${ }^{74}$. Thus, pharmacologic activation of HNF4 $\alpha$ may be useful in diseases affecting organs other than the liver. The HNF4 $\alpha$ activators studied here, NCT and NFT, differ in their respective abilities to activate the insulin promoter (Fig. 1). Thus, it may be desirable to screen for additional compounds to find those with optimal activity for each potential disease target.

\section{Materials and methods \\ Compound screening}

The T6PNE insulin promoter assay has been described previously ${ }^{10}$ and was performed here with slight modifications as follows: T6PNE cells were seeded at 2000 cells per well in 384-well tissue culture plates (Greiner Bio-One) in the presence of $0.5 \mu \mathrm{M}$ tamoxifen. Compounds listed in Fig. 1A in DMSO were dispensed with an Echo 555 Acoustic Liquid Handler (Beckman Coulter). Three days after compound addition, cells were fixed in 4\% paraformaldehyde (USBio) for $15 \mathrm{~min}$ and stained with DAPI $(0.167 \mu \mathrm{g} / \mathrm{ml}$, Invitrogen). Blue (DAPI) and green (GFP) channels were imaged using a Celigo imaging cytometer (Nexcelom Bioscience). The number of GFPpositive cells was normalized to the DAPI-positive cell number and fold change calculated relative to the DMSO control.

\section{Cell culture}

T6PNE cells were maintained in RPMI $(5.5 \mathrm{mM}$ glucose, Corning) supplemented with $10 \%$ fetal bovine serum (FBS, Sigma-Aldrich) and $1 \%$ penicillin-streptomycin (pen-strep, Gibco). Cells were maintained in $5 \% \mathrm{CO}_{2}$ at $37^{\circ} \mathrm{C}$. For the insulin promoter assay, $0.5 \mu \mathrm{M}$ tamoxifen (Sigma-Aldrich) was added to T6PNE cell culture media. HepG2 or HeLa cells were cultured in DMEM (high glucose, Corning) supplemented with 10\% FBS and 1\% pen-strep and maintained at $5 \% \mathrm{CO}_{2}, 37^{\circ} \mathrm{C}$.

\section{Lipid staining (in vitro) and analysis}

Oil red $\mathrm{O}$ and Nile Red staining were used to measure lipid accumulation. Oil Red $\mathrm{O}$ staining was done as described $^{75}$. Briefly, fixed cells were incubated with Oil Red $\mathrm{O}$ solution (Poly Scientific) for $3 \mathrm{~h}$, followed by photomicrography (Olympus, IX71). For Nile Red staining, dye (1: 500 in PBS from $1 \mathrm{mg} / \mathrm{ml}$ in ethanol stock, Sigma) was added for $30 \mathrm{mins}$ and DAPI added for 
10 mins at room temperature. Quantification of Nile Red staining was done with a Celigo imaging cytometer (Nexcelom Bioscience). More than 4000 cells were analyzed for each quantification. The number of Nile Redpositive cells was normalized to the DAPI-positive cell number for each well and fold change was calculated relative to a DMSO control well.

\section{Oil Red $\mathrm{O}$ staining (in vivo) and analysis}

Slides containing frozen liver tissue sections from mice were air dried for 10-20 min followed by rehydration in distilled water. Sections were immersed in absolute propylene glycol (Cat\# 151957, MP Biomedicals, LLC, USA) for $2 \mathrm{~min}$ followed by $0.5 \%$ in oil red $\mathrm{O}$ solution (Cat\#K043, Poly Scientific R\&D, USA) for $2 \mathrm{~h}$. Slides were then differentiated in $85 \%$ propylene glycol solution, washed with $\mathrm{dH}_{2} \mathrm{O}$ for $2 \mathrm{~h}$, and mounted using glycerin jelly mounting medium. All slides were scanned at a magnification of 20x using the Aperio Scanscope FL system (Aperio Technologies Inc., Vista, CA, USA). The liver area stained with oil red $\mathrm{O}$ was measured using image J software as described ${ }^{75}$, with some modifications. Oil red O-stained liver images were opened in Image J software. Using the Analyze $>$ Set Scale command, the scale bar of the images was set to $200 \mathrm{um}$. RGB images were then converted into gray scale images using the Image $>$ Type $>$ RGB Stack command and were split into red, blue and green channels. Using the Image $>$ Adjust $>$ Threshold command, the threshold was manually set to highlight the oil red O-stained lipid droplets in the green channel. We used the same threshold for all the images in all treatment groups and the $\%$ oil red O-stained area was obtained using the Analyze $\rightarrow$ Measure tool command. Fold change was calculated by normalizing the values to images from mice fed normal chow.

\section{Palmitate-BSA complex}

Palmitate $(150 \mathrm{mM})$ (Sigma-Aldrich) was prepared in $50 \%$ ethanol and precomplexed with 15\% fatty acid-free BSA (Research Organics, Cleveland, OH, USA) in a $37 \mathrm{C}$ water shaker. BSA-precomplexed palmitate was used as a $12 \mathrm{mM}$ stock solution for all assays with a final concentration of $0.25 \mathrm{mM}$ palmitate in cell culture medium.

\section{SiRNAs}

siRNAs were purchased from Ambion. For transfections, $24 \mu \mathrm{L}$ of each siRNA ( $1 \mu \mathrm{M}$ stock) was mixed with $90 \mu \mathrm{L}$ of a 1:100 dilution of Lipofectamine RNAi MAX (Invitrogen, Waltham, MA, USA) in Opti-MEM. Transfection was done in 24 well plates (Thermo Fisher Scientific, Waltham, MA, USA), by incubation with cells for $30 \mathrm{~min}$. at room temperature. One day after transfection, cells were transferred to 96 well plates (2000 cells per well) and incubated at $37^{\circ} \mathrm{C}$ for an additional day.
Forty-eight hours after transfection, either palmitate-BSA complex or BSA control plus or minus compounds was added for 2 days. For validation of siRNAs, transfected cells were harvested for RNA purification and QPCR for each gene 2 days after transfection.

\section{Q-PCR}

Quantitative PCR (Q-PCR) from in vitro cell culture experiments used total RNA purified using RNeasy Kits (Qiagen). For liver tissue samples, total RNA was isolated using Trizol (Invitrogen) and prepared for RNAseq (mouse liver DMSO and NCT, $N=3$ ). cDNA was amplified using $3 \mu \mathrm{g}$ of total RNA using qScript cDNA SuperMix (Quanta BioSciences, Beverly, MA, USA). QPCR analysis was performed using SYBR ${ }^{\circledR}$ Select Master Mix (Applied Biosystems) and ABI 7900HT (Applied Biosystems, Thermo Fisher Scientific). The Ct values of mRNA expression were then normalized to the $18 \mathrm{~s}$ rRNA values and are expressed as fold change over samples from mice fed normal chow for in vivo experiments or DMSO controls for cell culture experiments.

\section{DARTS assay}

This was conducted as described previously ${ }^{7}$. HepG2 cells were treated with DMSO, BI6015, NCT, NFT at a concentration of 40 or $80 \mu \mathrm{M}$ for $16 \mathrm{hr}$. Total cell protein was extracted and measured by BCA protein assay (Thermo Scientific). Each sample was split into two aliquots for proteolysis without $(-)$ or with $(+)$ Subtilisin (Sigma-Aldrich). Forty $\mathrm{mg}$ of cell lysate was incubated with or without protease $(40 \mathrm{ng} / \mathrm{ml}$ subtilisin) for $35 \mathrm{~min}$ at room temperature.

\section{Western blotting}

Whole-cell extracts were prepared by incubation in RIPA buffer (Invitrogen) containing protease inhibitors (Calbiochem, San Diego, CA). Protein $(40 \mathrm{mg})$ was separated on $12 \%$ or $16 \%$ Tri-Glycine gels (Invitrogen) and transferred to Immobilon $\mathrm{P}$ membrane $(0.2 \mu \mathrm{m}$ pore, Millipore). After $1 \mathrm{~h}$ in phosphate-buffered saline-Tween (PBST) with 3\% milk, membrane was incubated with antibodies to HNF4 $\alpha$ (mouse, Novus, 1:1000), LC3B (rabbit, Novus, 1:500), p62 (SQSTM1, mouse, Santa Cruz, 1:1000) or $\beta$-actin (mouse, Santa Cruz, 1:2000), followed by secondary antibody conjugated to horseradish peroxidase (1:5000, Jackson Immune). Signal was revealed by ECL (Thermo) and imaged with a ChemiDoc MP imager (Bio-Rad).

\section{BCA assay}

The bicinchoninic acid (BCA) protein assay kit assay (Thermo scientific) was used to measure protein amount for DART, Western blotting and TG assays. Absorbance at $550 \mathrm{~nm}$ was determined using a plate reader. 


\section{Mice}

12-week-old C57BL/6 J DIO male mice (cat\#380050) were purchased from Jackson Laboratory and were fed with high fat diet containing $60 \mathrm{kcal} \%$ fat (Research Diets cat \#D12492). Mice were maintained in a 12-h light/day cycle. After 2 weeks of acclimation, mice with similar body weights were randomly assigned to treatment or control groups.

For dose response experiments, mice were injected intraperitoneally with10\% DMSO as vehicle control or 4 different doses of NCT (30, 60, 120 and $240 \mathrm{mg} / \mathrm{kg}$ of body weight) dissolved in 10\% DMSO. Mice received 2 doses per day with a $5 \mathrm{~h}$ interval between injections for 3 days. Mice were observed for any adverse effects for a week of post treatment as shown in Supplementary Table 1 .

To test the effect of NCT (Sundia MediTech Company, Ltd., Custom synthesis), $200 \mathrm{mg} / \mathrm{kg}$ was injected IP bid for 14 days. On day 15, mice received a final dose of NCT followed by IP injection with $3 \mathrm{~g} / \mathrm{kg}$ dextrose. One h later, blood samples were collected and mice were euthanized using pentobarbital. Total mouse, liver and epididymal fat pad weights were measured. Dissected liver samples were washed in cold PBS, cut into small pieces and distributed for analyses. For RNA isolation and ELISA, liver samples were snap frozen using liquid nitrogen and stored at $-80^{\circ} \mathrm{C}$. For histomorphometry and immunofluorescence analysis, liver samples were fixed in $4 \%$ of cold PFA and processed for histology. All animal experiments were approved by the Institutional Animal Care and Use Committee (IACUC) of the Sanford Burnham Prebys Medical Discovery Institute in accordance with national regulations.

\section{Immunofluorescence and analysis}

Frozen liver sections were permeabilized using $0.3 \%$ Triton- $\mathrm{X}$ and incubated in antigen retrieval solution (Antigen retrieval citrate, Biogenex) at sub boiling temperature for $10 \mathrm{~min}$. Subsequently, sections were incubated with blocking buffer containing 5\% normal donkey serum (Jackson Immuno Research) followed by incubation overnight at $4{ }^{\circ} \mathrm{C}$ with mouse monoclonal primary antibody against HNF4 (1:800, Cat\# PP-H1415-00, R\&D Systems). Sections were washed and incubated with anti-mouse secondary antibody coupled with Alexa flour 488 (1:400, Invitrogen) or with DyLight 647 (1:400, Jackson Immuno) for $1 \mathrm{~h}$ at room temperature and counterstained with DAPI (40,6-diamidino2- phenylindole, Sigma Aldrich). For lipid droplet staining, slides were incubated in Bodipy 500/510 (1:100 from $1 \mathrm{mg}$ / $\mathrm{ml}$, 4,4-Difluoro-5-Methyl-4-Bora-3a,4a-Diaza-s-Indacene3-Dodecanoic Acid, Invitrogen) for $30 \mathrm{~min}$. Slides were mounted using fluorescence mounting medium and images were obtained at 40x magnification using an Olympus IX71 fluorescence microscope. Fluorescence intensity of
HNF4 $\alpha$-stained nuclei was calculated using MetaMorph TL software (version 7.6.5.0, Olympus).

\section{Free fatty acid quantification}

The serum FFA level was measured using the Free Fatty Acid Quantification Colorimetric/ Fluorometric Kit (Cat \#K612, BioVision, USA). Fold change was calculated by normalizing to values from mice fed normal chow.

\section{Triglyceride analysis}

Serum and liver TG level was measured using the Triglyceride Calorimetric Assay Kit (Cat\# 10010303, Cayman Chemicals, USA). Fold change was calculated by normalizing the values from normal chow mice.

\section{Liver profile analysis}

$100 \mu \mathrm{L}$ of whole blood was collected in lithium heparin blood collection tubes and transferred to single use VetScan mammalian liver profile reagent rotors. The levels of multiple analytes present in the blood samples were quantified using a VetScan VS2 Chemistry Analyzer (Abaxis North America, USA).

\section{Serum alkaline phosphatase analysis}

The alkaline phosphatase level in serum samples was quantified using a Catalyst One Chemistry Analyzer (IDEXX Laboratories, Inc. USA).

\section{Microsomal stability}

Microsomal stability studies were performed in the Conrad Prebys Center for Chemical Genomics. In vitro metabolism was conducted in a system consisted of NADPH generating system, test compound, and Tris. $\mathrm{Cl}$ buffer. The mixture was pre-incubated at $37^{\circ} \mathrm{C}$ for $30 \mathrm{~min}$. Reactions were initiated by addition of mouse or human liver microsomes suspension and shaken at $37^{\circ} \mathrm{C}$ with air exposure. To generate the stability curve for the test compound, the incubation was terminated at $0,5,15$, 30 and $60 \mathrm{~min}$. NFT and NCT concentrations were determined by LC-MS. The result of metabolic stability was expressed as the percentage of compound remaining at $1 \mathrm{hr}$. The in vitro half-life $(\mathrm{t} 1 / 2)$ and intrinsic clearance (Clint) were calculated based on drug depletion over incubation time.

\section{Pharmacokinetics}

Murine PK was conducted by WuXi AppTec (Shanghai, China). C57BL/6 male mice of age 7-9 weeks were obtained from SLAC Laboratory Animal Co (Shanghai, China). Mice were fasted for $12 \mathrm{~h}$ prior to compound administration. Oral gavage was used for PO dosing. For IV dosing, compound was administered by tail vein injection. For compound concentration determination, $25 \mu \mathrm{L}$ of blood was collected from the submandibular or 
saphenous vein and processed for plasma. Plasma concentration of compound was determined by LC-MS/MS.

\section{Lipidomics (Ceramide panel)}

Ceramides and dihydroceramides were measured in the UCSD Lipidomics Core Facility as previously described (Quehenberger et $\mathrm{al}^{54}{ }^{54}$. Briefly, samples were extracted using the butanol: methanol (BUME) method ${ }^{76}$. The lipid layer was collected and run on a Thermo-Vanquish UPLC (Thermo Scientific) with a Cortecs T3 (C18), $2.1 \mathrm{~mm} \times 150 \mathrm{~mm} ; 1.8 \mathrm{uT} 3$ column and a binary solvent system. Mass spectrometry employed a Thermo Q Exactive instrument with MS/MS data dependent acquisition scan mode and LipidSearch software (Thermo Fisher Scientific). Lipid nomenclature is given for the validated species: Cer d32:1_19.14|d18:1/n14:0. d stands for dihydroxy and $t$ sands for tri-hydroxy; d32:1 indicates that the sum total carbons is 32 and the species contains 1 double bond; the number following the underscore is the retention time; $\mathrm{d} 18: 1 / \mathrm{n} 14: 0$ indicates that indicates that the sphingoid base fatty acid is $18: 1$ and contains 2 hydroxy groups; $14: 0$ is the amide bonded fatty acid that contains no (n) hydroxy group; n14:0 indicates that 14:0 is the amide bonded fatty acid that contains no (n) hydroxy group.

\section{STRING network analysis}

STRING (https://string-db.org) shows protein-protein interaction networks. The top 50 gene candidates upregulated in NCT treated mouse liver $(N=3)$ were analyzed. STRING functional enrichment analysis was also performed.

\section{Primary human hepatocytes}

Experiments with primary human hepatocytes were performed by CN-Bio (Cambridge, UK). Primary human hepatocytes (PHHs), human Kupffer cells (HKs) and human stellate cells (HSCs) were seeded onto CN-Bio's PhysioMimix LC12 MPS culture plates at $6 \times 10^{5}$ cells for PHHs and $6 \times 10^{4}$ cells for HKs and HSCs in $1.6 \mathrm{ml}$ of CN-Bio's HEP-lean media with 5\% FCS. Throughout the experiment the cells were maintained at a flow rate of $1 \mu \mathrm{l} / \mathrm{s}$. After $24 \mathrm{~h}$ (Day 1) of seeding, the media was changed to HEP-lean media and the cells were incubated until day 4 to allow the cells to form microtissues. At day 4 post seeding, media was changed to HEP-fat media and treated with DMSO or $\operatorname{NCT}(5,15,40 \mu \mathrm{M})$. Media was replaced on days 6 and 8. Cells were harvested on day 10 for RNA extraction.

\section{Statistical analysis}

Data are presented as mean \pm SEM of three or more samples as indicated. Statistical significance was assessed using Student's $t$-test, ANOVA or $R^{2}$ coefficient of correlation.

\section{Reagents}

See Supplementary Table 6

\section{Acknowledgements}

We thank the following SBP Core Facilities: Imaging, Animal, Genomics, Conrad Prebys Center for Chemical Genomics, Histology. We thank the UCSD

Lipidomics Core. We thank Drs. Lee Chae and Jessie Ochoa of Brightseed for providing compounds and analytical chemistry support.

\section{Author contributions}

V.V. and S.-H.L. conducted research, analyzed data, and wrote the manuscript. F.L. was involved in conceptualization, data analysis, writing, and supervision.

\section{Funding}

This work was funded by the Sanford Children's Health Research Center and Brightseed, Inc.

\section{Data and materials availability}

Unique reagents generated in this study will be made available upon request. An agreement with our institute's Materials Transfer Agreement (MTA) may be required. Further information and requests for resources and reagents should be directed to Fred Levine (flevine@sbpdiscovery.org).

\section{Ethics}

No human subjects were used.

\section{Conflict of interest}

The authors declare no competing interests.

\section{Publisher's note}

Springer Nature remains neutral with regard to jurisdictional claims in published maps and institutional affiliations.

Supplementary information The online version contains supplementary material available at https://doi.org/10.1038/s41419-021-03862-x.

Received: 1 March 2021 Revised: 18 May 2021 Accepted: 21 May 2021 Published online: 11 June 2021

\section{References}

1. Friedman, S. L., Neuschwander-Tetri, B. A., Rinella, M. \& Sanyal, A. J. Mechanisms of NAFLD development and therapeutic strategies. Nat. Med 24, 908-22 (2018).

2. Samji, N. S., Verma, R. \& Satapathy, S. K. Magnitude of nonalcoholic fatty liver disease: western perspective. J. Clin. Exp. Hepatol. 9, 497-505 (2019).

3. Hagström, H., Thiele, M., Roelstraete, B., Söderling, J. \& Ludvigsson, J. F. Mortality in biopsy-proven alcohol-related liver disease: a population-based nationwide cohort study of 3453 patients. Gut 70, 170-179 https:/doi.org/ 10.1136/gutjnl-2019-320446 (2021).

4. Fruhbeck, G., Mendez-Gimenez, L., Fernandez-Formoso, J. A., Fernandez, S. \& Rodriguez, A. Regulation of adipocyte lipolysis. Nutr. Res Rev. 27, 63-93 (2014).

5. Rui, L. Energy metabolism in the liver. Compr. Physiol. 4, 177-97 (2014).

6. Gluchowski, N. L., Becuwe, M., Walther, T. C. \& Farese, R. V. Jr. Lipid droplets and liver disease: from basic biology to clinical implications. Nat. Rev. Gastroenterol. Hepatol. 14, 343-55 (2017).

7. Lee, S. H. et al. Identification of alverine and benfluorex as HNF4alpha activators. ACS Chem. Biol. 8, 1730-6 (2013).

8. Kiselyuk, A. et al. HNF4alpha antagonists discovered by a high-throughput screen for modulators of the human insulin promoter. Chem. Biol. 19, 806-18 (2012).

9. Cohen T., Sundaresh S. \& Levine F. Antipsychotics activate the TGFbeta pathway effector SMAD3. Mol. Psychiatry 18, 347-357 (2012).

10. Kiselyuk, A. et al. Phenothiazine neuroleptics signal to the human insulin promoter as revealed by a novel high-throughput screen. J. Biomol. Screen 15, 663-70 (2010).

11. Kiselyuk, A. et al. High-throughput screening for small molecules that regulate insulin promoter activity. Diabetes 56, A430 (2007). 
12. Hwang-Verslues, W. W. \& Sladek, F. M. HNF4alpha-role in drug metabolism and potential drug target? Curr. Opin. Pharmacol. 10, 698-705 (2010).

13. Martinez-Jimenez, C. P., Kyrmizi, I., Cardot, P., Gonzalez, F. J. \& Talianidis, I. Hepatocyte nuclear factor 4alpha coordinates a transcription factor network regulating hepatic fatty acid metabolism. Mol. Cell Biol. 30, 565-77 (2009).

14. Yuan, $X$. et al. Identification of an endogenous ligand bound to a native orphan nuclear receptor. PLOS ONE 4, e5609 (2009).

15. Dhe-Paganon, S., Duda, K., Iwamoto, M., Chi, Y. I. \& Shoelson, S. E. Crystal structure of the HNF4 alpha ligand binding domain in complex with endogenous fatty acid ligand. J. Biol. Chem. 277, 37973-6 (2002).

16. Moulin, P. et al. Efficacy of benfluorex in combination with sulfonylurea in type 2 diabetic patients: an 18-week, randomized, double-blind study. Diabetes Care 29, 515-20 (2006)

17. Del Prato, S., Erkelens, D. W. \& Leutenegger, M. Six-month efficacy of benfluorex vs. placebo or metformin in diet-failed type 2 diabetic patients. Acta Diabetol. 40, 20-7 (2003)

18. Facchini, P. J., Hagel, J. \& Zulak, K. G. Hydroxycinnamic acid amide metabolism: physiology and biochemistry. Can. J. Bot. 80, 577-89 (2002).

19. Bernards M. A. \& Båstrup-Spohr L. Phenylpropanoid metabolism induced by wounding and insect herbivory. In Induced Plant Resistance to Herbivory (ed. Schaller A.) 189-211 (Dordrecht, Springer Netherlands, 2008).

20. Kounakis, K., Chaniotakis, M., Markaki, M. \& Tavernarakis, N. Emerging roles of lipophagy in health and disease. Front Cell Dev. Biol. 7, 185 (2019).

21. Huang, P., Chandra, V. \& Rastinejad, F. Structural overview of the nuclear receptor superfamily: insights into physiology and therapeutics. Annu. Rev. Physiol. 72, 247-72 (2010).

22. Bailly, A., Torres-Padilla, M. E., Tinel, A. P. \& Weiss, M. C. An enhancer element 6 $\mathrm{kb}$ upstream of the mouse HNF4alpha1 promoter is activated by glucocorticoids and liver-enriched transcription factors. Nucleic Acid Res. 29, 3495-505 (2001).

23. Bolotin, E. et al. Integrated approach for the identification of human hepatocyte nuclear factor 4 alpha target genes using protein binding microarrays. Hepatology 51, 642-53 (2010).

24. Lomenick, B. et al. Target identification using drug affinity responsive target stability (DARTS). Proc. Natl Acad. Sci. USA 106, 21984-9 (2009).

25. Bonzo, J. A., Ferry, C. H., Matsubara, T., Kim, J. H. \& Gonzalez, F. J. Suppression of hepatocyte proliferation by hepatocyte nuclear factor 4alpha in adult mice. J. Biol. Chem. 287, 7345-56 (2012).

26. Carta, G., Murru, E., Banni, S. \& Manca, C. Palmitic acid: physiological role, metabolism and nutritional implications. Front Physiol. 8, 902 (2017).

27. Wigger, D., Gulbins, E., Kleuser, B. \& Schumacher, F. Monitoring the sphingolipid de novo synthesis by stable-isotope labeling and liquid chromatographymass spectrometry. Front Cell Dev. Biol. 7, 210 (2019).

28. Scott, L. J. Fingolimod: a review of its use in the management of relapsingremitting multiple sclerosis. CNS Drugs 25, 673-98 (2011).

29. Osawa, Y. et al. Sphingosine kinase regulates hepatoma cell differentiation: roles of hepatocyte nuclear factor and retinoid receptor. Biochem. Biophys. Res. Commun. 286, 673-7 (2001).

30. Blaho, V. A. \& Hla, T. An update on the biology of sphingosine 1-phosphate receptors. J. lipid Res. 55, 1596-608 (2014).

31. Nagahashi, M. et al. Spns2, a transporter of phosphorylated sphingoid bases, regulates their blood and lymph levels, and the lymphatic network. FASEB $J$. 27, 1001-11 (2013).

32. Bikman, B. T. et al. Fenretinide prevents lipid-induced insulin resistance by blocking ceramide biosynthesis. J. Biol. Chem. 287, 17426-37 (2012).

33. Mody, N. \& Mcllroy, G. D. The mechanisms of Fenretinide-mediated anticancer activity and prevention of obesity and type-2 diabetes. Biochem. Pharm. 91, 277-86 (2014).

34. Siddique, M. M., Li, Y., Chaurasia, B., Kaddai, V. A. \& Summers, S. A. Dihydroceramides: from bit players to lead actors. J. Biol. Chem. 290, 15371-9 (2015).

35. Gottlieb, R. A., Andres, A. M., Sin, J. \& Taylor, D. P. Untangling autophagy measurements: all fluxed up. Circ. Res. 116, 504-14 (2015).

36. Sahani, M. H., Itakura, E. \& Mizushima, N. Expression of the autophagy substrate SQSTM1/p62 is restored during prolonged starvation depending on transcriptional upregulation and autophagy-derived amino acids. Autophagy 10, 431-41 (2014).

37. Ko, J. H., Yoon, S. O., Lee, H. J. \& Oh, J. Y. Rapamycin regulates macrophage activation by inhibiting NLRP3 inflammasome-p38 MAPK-NFkappaB pathways in autophagy- and p62-dependent manners. Oncotarget 8, 40817-31 (2017).
38. Schulze, R. J. et al. Direct lysosome-based autophagy of lipid droplets in hepatocytes. Proc. Natl Acad. Sci. USA 117, 32443-52 (2020).

39. Rosenbaum, A. I. et al. Chemical screen to reduce sterol accumulation in Niemann-Pick $C$ disease cells identifies novel lysosomal acid lipase inhibitors. Biochim. Biophys. Acta 1791, 1155-65 (2009).

40. Huang, K. W. et al. Liver activation of hepatocellular nuclear factor-4alpha by small activating RNA rescues dyslipidemia and improves metabolic profile. Mol. Ther. Nucleic Acids 19, 361-70 (2020).

41. Collins S. M., et al. Free fatty acids as an indicator of the nonfasted state in children. Pediatrics 143, e20183896 https://doi.org/10.1542/peds.2018-3896 (2019).

42. Baker, N., Hill, V. \& Ookhtens, M. Regulation of plasma-free fatty acid mobilization by dietary glucose in Ehrlich ascites tumor-bearing mice. Cancer Res. $\mathbf{3 8}$ 2372-7 (1978).

43. Kocabay, G. et al. Alkaline phosphatase: can it be considered as an indicator of liver fibrosis in non-alcoholic steatohepatitis with type 2 diabetes? Bratisl. Lek. Listy 112, 626-9 (2011).

44. Tahan, V. et al. Serum gamma-glutamyltranspeptidase distinguishes nonalcoholic fatty liver disease at high risk. Hepatogastroenterology 55, 1433-8 (2008).

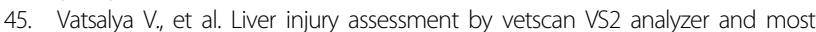
frequently used ALT/GTP reagent. Gastroenterol Hepatol. 4, 107 (2016).

46. Boj, S. F., Parrizas, M., Maestro, M. A. \& Ferrer, J. A transcription factor regulatory circuit in differentiated pancreatic cells. Proc. Natl Acad. Sci. USA 98, 14481-6 (2001).

47. Spath, G. F. \& Weiss, M. C. Hepatocyte nuclear factor 4 provokes expression of epithelial marker genes, acting as a morphogen in dedifferentiated hepatoma cells. J. Cell Biol. 140, 935-46 (1998).

48. Chellappa, K. et al. Src tyrosine kinase phosphorylation of nuclear receptor HNF4alpha correlates with isoform-specific loss of HNF4alpha in human colon cancer. Proc. Natl Acad. Sci. USA 109, 2302-7 (2012).

49. Zolfaghari, R. \& Ross, A. C. Hepatocyte nuclear factor 4alpha (HNF4alpha) in coordination with retinoic acid receptors increases all-trans-retinoic aciddependent CYP26A1 gene expression in HepG2 human hepatocytes. J. Cell Biochem. 115, 1740-51 (2014).

50. Chithalen, J. V., Luu, L., Petkovich, M. \& Jones, G. HPLC-MS/MS analysis of the products generated from all-trans-retinoic acid using recombinant human CYP26A. J. Lipid Res. 43, 1133-42 (2002)

51. Kedishvili, N. Y. Retinoic acid synthesis and degradation. Subcell. Biochem. 81, 127-61 (2016).

52. Yang, K., Koh, K. H. \& Jeong, H. Induction of CYP2B6 and CYP3A4 expression by 1-aminobenzotriazole (ABT) in human hepatocytes. Drug Metab. Lett. 4, 129-33 (2010).

53. Stevison, F., Hogarth, C., Tripathy, S., Kent, T. \& Isoherranen, N. Inhibition of the all-trans retinoic acid (atRA) hydroxylases CYP26A1 and CYP26B1 results in dynamic, tissue-specific changes in endogenous atRA signaling. Drug Metab. Dispos. 45, 846-54. (2017).

54. Quehenberger, $\mathrm{O}$. et al. Lipidomics reveals a remarkable diversity of lipids in human plasma. J. Lipid Res. 51, 3299-305 (2010).

55. Cristoferi, L. et al. Prognostic models in primary biliary cholangitis. J. Autoimmun. 95, 171-8 (2018).

56. Kowdley, K. V. et al. A randomized trial of obeticholic acid monotherapy in patients with primary biliary cholangitis. Hepatology 67, 1890-902 (2018).

57. Guy, R. K., DiPaola, R. S., Romanelli, F. \& Dutch, R. E. Rapid repurposing of drugs for COVID-19. Science 368, 829-30 (2020).

58. Jourdan, J. P., Bureau, R., Rochais, C. \& Dallemagne, P. Drug repositioning: a brief overview. J. Pharm. Pharm. 72, 1145-51 (2020).

59. Islam M. T., Ali E. S., Mubarak M. S. Anti-obesity effect of plant diterpenes and their derivatives: a review. Phytother. Res. 34, 1216 (2020).

60. Oguntibeju, O. O. Hypoglycaemic and anti-diabetic activity of selected African medicinal plants. Int. J. Physiol. Pathophysiol. Pharm. 11, 224-37 (2019).

61. Jiang, Y., Yu, L. \& Wang, M. H. N-trans-feruloyltyramine inhibits LPS-induced NO and PGE2 production in RAW 264.7 macrophages: Involvement of AP1 and MAP kinase signalling pathways. Chem. Biol. Interact. 235, 56-62 (2015).

62. Tang J., Dunshea F. R., Suleria H. A. R. LC-ESI-QTOF/MS characterization of phenolic compounds from medicinal plants (Hops and Juniper Berries) and their antioxidant activity. Foods 9, 7 (2019).

63. Mao, Y., Yu, F., Wang, J., Guo, C. \& Fan, X. Autophagy: a new target for nonalcoholic fatty liver disease therapy. Hepat. Med. 8, 27-37 (2016). 
64. Schmeisser, K.\& Parker, J. A. Pleiotropic effects of mTOR and autophagy during development and aging. Front. Cell Dev. Biol. 7, 192 (2019).

65. Isoherranen, N. \& Zhong, G. Biochemical and physiological importance of the CYP26 retinoic acid hydroxylases. Pharmacol. therapeutics 204, 107400 (2019).

66. Wigger, L. et al. Plasma dihydroceramides are diabetes susceptibility biomarker candidates in mice and humans. Cell Rep. 18, 2269-79 (2017).

67. Chaurasia, B. et al. Targeting a ceramide double bond improves insulin resistance and hepatic steatosis. Science 365, 386-92 (2019).

68. Xie, S. Z. et al. Sphingolipid modulation activates proteostasis programs to govern human hematopoietic stem cell self-renewal. Cell Stem Cell 25, 639-53 e7 (2019)

69. Lau, H. H., Ng, N. H. J., Loo, L. S. W., Jasmen, J. B. \& Teo, A. K. K. The molecular functions of hepatocyte nuclear factors - In and beyond the liver. J. Hepatol. 68, 1033-48 (2018).

70. Mohlke, K. L. \& Boehnke, M. The role of HNF4A variants in the risk of type 2 diabetes. Curr. Diabetes Rep. 5, 149-56 (2005).
71. Yamagata, K. et al. Mutations in the hepatocyte nuclear factor-4alpha gene in maturity-onset diabetes of the young (MODY1). Nature 384, 458-60 (1996).

72. $\mathrm{Mu}, \mathrm{W}$. et al. Potential nexus of non-alcoholic fatty liver disease and type 2 diabetes mellitus: insulin resistance between hepatic and peripheral tissues. Front Pharm. 9, 1566 (2018).

73. Barrett, J. C. et al. Genome-wide association study of ulcerative colitis identifies three new susceptibility loci, including the HNF4A region. Nat. Genet $\mathbf{4 1}$, 1330-4 (2009).

74. Kovesdy, C. P., Furth, S. L. \& Zoccali, C. World kidney day steering C. Obesity and kidney disease: hidden consequences of the epidemic. Can. J. Kidney Health Dis. 4, 2054358117698669 (2017)

75. Mehlem, A., Hagberg, C. E., Muhl, L., Eriksson, U. \& Falkevall, A. Imaging of neutral lipids by oil red $\mathrm{O}$ for analyzing the metabolic status in health and disease. Nat. Protoc. 8, 1149-54 (2013).

76. Lofgren, L., Forsberg, G. B. \& Stahlman, M. The BUME method: a new rapid and simple chloroform-free method for total lipid extraction of animal tissue. Sci. Rep. 6, 27688 (2016) 